UDC 517.9

\title{
A COMPLETE ASYMPTOTIC ANALYSIS OF AN OSCILLATION FREE NONLINEAR EQUATION OF BESSEL TYPE WITH A POLE IN THE DEPENDENT VARIABLE

ПОВНИЙ АСИМПТОТИЧНИЙ АНАЛІЗ НЕЛІНІЙНОГО РІВНЯННЯ
БЕССЕЛЕВСЬКОГО ТИПУ БЕЗ КОЛИВАНЬ
3 ПОЛЮСОМ ВІДНОСНО ЗАЛЕЖНОЇ ЗМІННОЇ

A. B. Mingarelli

School Math. and Statistics

Carleton Univ.

Ottawa, Ontario, Canada, K1S 5B6

e-mail: amingare@math.carleton.ca

\section{J. M. Pacheco-Castelao}

Univ. Las Palmas de Gran Canaria

Campus de Tafira Baja

35017 Las Palmas de Gran Canaria, Spain

e-mail: pacheco@dma.ulpgc.es

\section{S. Melkonian}

School Math. and Statistics

Carleton Univ.

Ottawa, Ontario, Canada, K1S 5B6

e-mail:melkonia@math.carleton.ca

\begin{abstract}
We characterize the solution set of a nonlinear perturbation of Bessel's equation of order zero on a halfline where the nonlinearity is analytic in the independent variable, algebraic in the dependent variable and, indeed, admits a pole in this variable. We show that the equation fails the Painleve test and that there are no points in $[0, \infty)$ where blow-up occurs. Although we cannot find even one closed-form solution, it is shown that there are only four families of solutions: those that are asymptotically linear and increasing, solutions that are asymptotically linear and decreasing, another set of solutions that are asymptotically constant, and a final set of solutions that admit singularities at finite points on $[0, \infty)$. As a consequence, we deduce that every solution with or without singularities on $[0, \infty)$ is non-oscillatory and, in fact, has at most two zeros. We also show that the plane $\Pi$ of real initial conditions $\left(y(0), y^{\prime}(0)\right)$ can be decomposed into a union of connected regions, in each of which the solutions are exactly one of the types mentioned above. Furthermore, we obtain that the set of those initial conditions leading to asymptotically constant solutions is a piecewise differentiable curve in $\Pi$, one that can be estimated theoretically to a high degree of precision. In addition, the asymptotic behavior of solutions near a finite singularity is obtained. Estimates relating the growth of solutions to their initial conditions are also described and numerical examples are presented to illustrate the theory. Finally, we observe that every solution of our equation has finite singularities when viewed as a solution on the whole line.
\end{abstract}

Наведено опис множини розв'язків нелінійно збуреного рівняння Бесселя нульового порядку на півосі, де нелінійність є аналітичною відносно незалежної змінної, алгебраїчною відносно залеж-

(C) A. B. Mingarelli, J. M. Pacheco-Castelao, S. Melkonian, 2010

ISSN 1562-3076. Нелінійні коливання, 2010, m. 13, N으 
ної змінної та фактично мае полюс за цією змінною. Показано, щцо рівняння не задовольняе ознаки Пейнлеве та не існує точок на $[0, \infty)$, де розв'язок прямує до нескінченності. I хоча не було знайдено розв'язку в явній формі, доведено, щзо існують лише чотири сім'ї розв'язків: асимптотично лінійних та зростаючих, асимптотично лінійних та спадних, асимптотично сталих та остання множина розв'язків, які можуть мати особливості в скінченних точках $[0, \infty)$. Як наслідок встановлено, щзо кожний розв'язок, щзо має або не має особливості, є неколивним і рактично має не більще двох нулів. Також показано, щзо площину П дійсних початкових умов $\left(y(0), y^{\prime}(0)\right)$ можна розбити на об'єднання зв'язних множин, в кожній з яких розв'язок належить одній з описаних вище множин. Доведено, що множина початкових умов, які приводять до асимптотично сталих розв'язків, є кусково-диференційовною кривою в П $і$ може бути оцінена з високою точністю. Також описано асимптотичну поведінку розв'язків в околі скінченної особливості. Отримано оцінки зростання розв'язків в залежності від початкових умов і наведено числові приклади, які ілюструють теорію. Насамкінець показано, щзо кожен розв'язок рівняння має скінченні особливості, якщо розглядати його на всій прямій.

1. Introduction. Motivated by a recent paper of Dubé-Mingarelli [10], we present an in-depth study of the final example in [10] dealing with the existence of asymptotically constant solutions of the nonlinear differential equation

$$
y^{\prime \prime}+\frac{e^{-x}}{4} y+\frac{e^{-2 x}}{1+y}=0, \quad x \in[0, \infty)
$$

where we note the possible presence of a singularity at a point $x_{0}$ where $y\left(x_{0}\right)=-1$. By a classical solution of (1) on $[0, \infty)$ we mean a function that is of class $C^{2}(0, \infty)$ which satisfies the equation everywhere. First of all, we note that (1) is actually a nonlinear perturbation of a Bessel equation of order zero in disguise. That is, the transformation $t=e^{-x / 2}, y(x)=z(t)$, transforms (1) to an equation of the form

$$
t^{2} z^{\prime \prime}+t z^{\prime}+t^{2} z+\frac{4 t^{4}}{1+z}=0, \quad t \in(0,1]
$$

Our results for (1) will therefore apply to this equation (2) upon consideration of the quantity $z(t)=y\left(\ln \left(t^{-2}\right)\right)$.

The formulation of general criteria for the existence of positive monotone solutions of second order nonlinear real differential equations of the form $y^{\prime \prime}(x)+F(x, y(x))=0$, defined on $[0, \infty)$, has been a subject of current interest. Methods used vary from using the fixed point theorem of Banach-Cacciopoli, Hale [12], to Schauder's fixed point theorem (see [12]), and other similar methods. The nonlinearity, being of the form $F(x, y(x))$, is not assumed to be variables-separable, nor is it assumed that estimates of the form $|F(x, y)| \leq f(x) g(y)$ are in force as is usually the case in studies of this character, cf., Constantin [9], Nasr [21], Yin [28], Zhao [31]. In the cited papers, one finds criteria for the existence of at least one positive solution of a class of equations with various properties, as opposed to here, where we actually find a description of every possible solution of (1), positive or not. Next, we note that the asymptotic methods of Hartman, Onuchic [14] and Hale, Onuchic [13] are not applicable here because of the presence of the pole in the dependent variable, that is, given a real second order nonlinear differential equation of the form (in the notation of [14])

$$
u^{\prime \prime}=h\left(x, u, u^{\prime}\right),
$$

ISSN 1562-3076. Нелінійні коливання, 2010, m. 13, №2 
one normally requires the continuity of $h$ as a function of the variable $u$ for $u \in \mathbb{R}$, an assumption that is clearly not satisfied for our equation (1). Modern techniques require, at the very least, local integrability of $h$ as a function of $u$, so that, once again, this fails.

The origin of Eq. (1) in Dubé, Mingarelli [10] is summarized next. In order to describe the novelty and applicability of the results obtained in [10], one would need to find an equation for which most, if not all, existing non-oscillation criteria would not be applicable. In order to do this, one would need to construct an equation with at least three terms in order to circumvent the variables-separable case of $F$ mentioned earlier. On the other hand, and to the best of our knowledge, no existing criterion applies in the case where a pole in the dependent variable is introduced. The two additional exponential terms in (1) were introduced in order to satisfy an integrability condition on both our function $F$, as a function of its second variable, and a function $k(t)$, leading to a generalized Lipschitz condition on $F$ of Perron-Kamke-type (cf., [10]). The resulting equation became a nonlinear perturbation of a second-order (non-oscillatory) linear equation with a nonlinearity having a pole in the dependent variable. As a whole, the resulting equation (i.e., (1)) turned out to be one which is rational in the dependent variables and analytic in the independent variable so that, on the surface, Painlevé theory may be applicable. However, such is not the case, as we show in the discussion below, since (1) fails the Painlevé Test (cf., Ablowitz et al. [1], Ince [16]). Thus, the equation has movable critical points.

We present a brief non-exhaustive review of second-order equations with singularities that are poles in the dependent variable. In the event that the equation is analytic in its independent variable and rational in its dependent variable, this includes the Painlevé equations (50 of them) and the resulting theory (cf., [16], Chapter 14) which, in the end, may be reduced to the study of six specific equations (denoted consecutively by $P_{I}, P_{I I}, \ldots, P_{V I}$ ). The study of these six Painlevé equations has gathered momentum in the past 20 years with the monograph of Ablowitz and Clarkson [1] and others and their applicability to inverse scattering, physics, etc. Their importance lies in part with the fact that their general solutions produce new transcendents, that is, solutions that cannot be expressed in terms of known transcendental functions, including the classical special functions of mathematical physics (Bessel, Airy, Hypergeometric, etc.). The fact that a second-order equation (such as (1)) can fail the Painlevé test for the non-existence of movable critical points is of interest since we must then apply methods specific to the equation itself in order to obtain information about its general solution.

Besides this special class of second-order equations bearing Painlevé's name, we find in the literature a study of special classes of nonlinear singular integral equations (albeit on a finite interval) where the singularities are possible poles in the dependent variable. In the event that the kernel is replaced by a suitable Green's function, we see that such equations can include second-order equations with similar nonlinearities as a special case. One of the earliest papers in this respect is Nowosad [22] where results from doubly-stochastic matrices (see also Michelli, Olsen [22]) are used, in particular, to detect the existence of continuous positive solutions on a finite interval of equations of the form

$$
\bar{F}(x) \int_{0}^{1} K(x, y) F(y) d y=1, \quad 0 \leq x \leq 1
$$

where $F$ is the Fourier transform of a complex-valued function $f \in L^{2}(\mathbb{R})$ and $K$ is a positive kernel with additional properties (cf., Nowosad [22] for more details). An extension of this 
result to equations of the form

$$
v(s)=\int_{0}^{1} K(s, t) \frac{1}{v(t)^{\alpha}} d t, \quad 0 \leq s \leq 1,
$$

is considered in Karlin, Nirenberg [18] where $\alpha>0$, with a view to obtaining the existence of a continuous positive solution $v(t), t \in[0,1]$. However, the possibility that (4) may also admit solutions with movable zeros, i.e., points $x_{0}>0$ where $v\left(x_{0}\right)=0$, is not entertained. A study of Volterra and Fredholm operators $K$ on $\mathcal{C}[0,1]$ appears in Bushell [8] with an eye to obtaining the existence (and uniqueness) of positive solutions of integral equations of the form $(I-K) x^{p}=f$, where $-1<p<1$, thus allowing for the possibility of a singularity in the dependent variable, $x$, in the nature of a branch point. An extension of the results in [18] can be found in the articles by Parker, Walters [26], [29], once again relating the problem to fundamental results from matrix theory involving the similarity of an irreducible nonnegative square matrix to a doubly-stochastic matrix (Hartfiel's problem, see [26]). We would not be led too far astray if we were to mention that a significant development of the theory arising from further studies of Nowosad [22] and Karlin, Nirenberg [18] lies in the connection with the socalled DAD problem, once again motivated by matrix theory (cf., Nussbaum [23], [24], [25] and Borwein et al. [7]). More recently, a two-point boundary value problem on a finite interval in boundary layer theory appeared in Wang et al. [30] where, once again, the nonlinarity involves a pole in the dependent variable, and the problem addresses the question of the existence and non-existence of positive solutions on the finite interval. In addition, more direct extensions of the main results in [18] are also considered in [3], [4] and [5], although the authors appear not to be aware of the extensions of [18] via the DAD problem mentioned earlier.

For partial differential equations with singularities of the type we seek, we note Gomes [11] where the Dirichlet problem for $L u=k(x) u^{-\alpha}, \alpha>0$, and $L$ is elliptic, is considered on a bounded region in $\mathbb{R}^{N}, N \geq 3$, with a view at obtaining the existence of a positive solution. A result complementing Gomes [11] appears in Jin [17] where, although the nonlinearity is more general than an inverse power of the dependent variable, the operator $L$ is now the Laplacian on $\mathbb{R}^{n}, n \geq 3$. In addition, Jin [17] considers the Dirichlet problem on a finite domain or, in case the domain is all of $\mathbf{R}^{n}$, the solution sought is to be positive, as usual. In the same direction we find the work of Alves et al. [6] that falls in between Gomes [11] and Jin [17] by allowing a linear perturbation of the Laplacian and a nonlinearity of the type considered by Gomes [11].

In this paper, we consider the problem of determining the asymptotics of every solution of (1). There are two possible approaches to determining the long-term behavior of these solutions of our equation; the first is to consider (1) as a perturbation of a Bessel equation as we have shown above. We can then use the method of variation of parameters and estimate the integrals using known asymptotics of Bessel functions under some assumptions on the nonlinearity. On the other hand, and this is the method we prefer, we use more analytical methods, which include fixed point theorems and Lyapunov functions, thereby allowing for further generality in the scope of the equations that can be studied.

Although (1) is basically a simple-looking differential equation, we will see that it has a rich structure, a structure that does not appear to have been studied in the literature in such generality. In this case, the plane $\Pi$ of real initial conditions is divided into three connected regions separated by a piecewise differentiable curve along which initial data lead to asymptotically 
constant solutions. Another piece consists of initial data which produce solutions with finite singularities, while the remaining part gives asymptotically linear solutions. In Section 2, we initiate a study of the qualitative behavior of solutions by defining, in the first instance, a Lyapunov function that will be used extensively in the sequel. In Subsection 2.1 we connect (1) to the Painlevé program and show that it fails both the Painlevé and the Ablowitz-Ramani-Segur tests, thereby indicating the existence of movable critical points (which manifest themselves as branch points in our case). We also show that finite blow-up points do not exist for a given solution $y$ of (1), but that both derivatives can and do blow up at finite points, the location of which depends upon the particular solution considered.

After introducing the notions of non-oscillation and disconjugacy in Subsection 2.2, we show that all non-negative solutions are actually disconjugate (i.e., have at most one zero in $[0, \infty)$ ). Section 3 is devoted to describing the complete set of solutions of (1) (with or without singularities on $[0, \infty)$ ) via their limits at infinity. In Subsection 3.1, we show that the solutions obtained in Section 3 are either asymptotically linear and increasing, asymptotically constant, asymptotically linear and decreasing or, finally, solutions with finite blow-up in the first and second derivatives (finite singularities). We also describe methods of finding them numerically via the contraction mapping principle. Finally, in Section 4, we connect the solutions of (1) to their initial conditions and show how to find the initial conditions given the asymptotics and vice-versa. In addition, we show that the plane $\Pi$ of real initial conditions $\left(y(0), y^{\prime}(0)\right)$ can be decomposed into a union of connected regions, in each one of which, the solutions are exactly one of the types mentioned above. Furthermore, we obtain that the set of those initial conditions leading to asymptotically constant solutions is a piecewise differentiable curve in $\Pi$, one that can be estimated theoretically to a high degree of precision. Estimates relating the growth of solutions to their initial conditions are also described and numerical examples are presented to illustrate the theory.

2. The qualitative behavior of solutions. We state here the required motivating result from Dubé, Mingarelli [10] for reference purposes.

Theorem 1 (see Example 3.5 in [10]). Equation (1) admits a positive (on [0, $\infty)$ ) monotone solution that is asymptotic to one as $x \rightarrow \infty$.

We propose to analyze the remaining solutions of (1) including those with finite singularities. To this end we use the following notation. The initial conditions are assumed to be real and given by $y(0) \equiv y_{0}$, and $y^{\prime}(0) \equiv y_{0}^{\prime}$ and $y(a) \equiv y_{a}$, and $y^{\prime}(a) \equiv y_{a}^{\prime}$ where $a>0$ when appropriate. The fundamental existence and uniqueness theorem guarantees the existence of local solutions of (1) on some small interval $[0, \delta]$, where $\delta>0$, until a finite singularity is encountered (which generally moves with changes in the initial conditions). In this case the solution ceases to exist in a classical sense. Any interval $\mathcal{I} \subset[0, \infty)$ encountered in the sequel is assumed to be non-empty.

Remark 1. Note that there are three possible types of solution to equation (1): 1) solutions $y$ for which $y(x)>-1$ for all $x \in[0, \infty) ; 2$ ) solutions for which $y(x)<-1$ for all $x \in[0, \infty)$; $3)$ solutions for which there is a first point $x_{0} \in[0, \infty)$, generally depending on the initial conditions, such that $y\left(x_{0}\right)=-1$ (their existence is considered in Subsection 2.1).

Lemma 1. Let $y$ denote a solution of (1) defined on an interval $[a, x]$, where $a \geq 0$ and $x>a$. 
Then there holds the identity

$$
\frac{1}{2} y^{\prime}(x)^{2}+\frac{1}{8} e^{-x} y(x)^{2}+e^{-2 x} \ln |1+y(x)|+\frac{1}{8} \int_{a}^{x} e^{-t} y(t)^{2} d t+2 \int_{a}^{x} e^{-2 t} \ln |1+y(t)| d t \equiv I_{a},
$$

where

$$
I_{a}=\frac{1}{2} y_{a}^{\prime 2}+\frac{1}{8} e^{-a} y_{a}^{2}+e^{-2 a} \ln \left|1+y_{a}\right|
$$

The implicit relation (6) defines a curve in $\Pi:\left(y_{a}, y_{a}^{\prime}\right)$. The case of interest here is where a $=0$, in which case this curve is bell-shaped and opens to the left. When there is no confusion we will denote $I_{0}$ simply by $I$, for brevity.

Proof. We multiply (1) by $y^{\prime}(x)$ and integrate over the interval $[a, x]$.

The next results follow immediately from (5), thereby defining a Lyapunov function on nonnegative solutions.

Corollary 1. If $y$ is any non-negative solution of (1) on $[a, x]$, where $a \geq 0$, the function

$$
H(x)=\frac{1}{2} y^{\prime}(x)^{2}+\frac{1}{8} e^{-x} y(x)^{2}+e^{-2 x} \ln |1+y(x)|
$$

is non-negative and non-increasing.

As a result, we obtain the following corollary.

Corollary 2. For any non-negative solution y of (1) over $[a, x]$, we have

$$
\frac{1}{2} y^{\prime}(x)^{2}+\frac{1}{8} e^{-x} y(x)^{2}+e^{-2 x} \ln |1+y(x)| \leq I_{a}
$$

Lemma 2. Choose a pair of initial conditions $\left(y_{0}, y_{0}^{\prime}\right)$ such that $I \leq 0$. Then the corresponding solution $y(x) \leq 0$ on any interval to the right of $x=0$ so long as it is defined there (along with its second derivative).

Proof. Note that $y \equiv 0$ is not a solution of (1). Thus, let $y(x)$ be defined on $[0, \delta]$ (along with its second derivative) and suppose, on the contrary, that $y(x) \geq 0$ there. Then using Lemma 1 with $a=0$ and $x=\delta$, we obtain an immediate contradiction.

In particular, we note that whenever $I \leq 0$ then $-2 \leq y_{0} \leq 0, y_{0} \neq-1$.

2.1. On the existence of movable critical points and movable singularities. We recall that a critical point of a differential equation is any singularity other than a pole (see Ince [16, p. 317] and Ablowitz, Clarkson [1, p. 348]). Such a critical point is said to be movable if its location depends upon the choice of the initial conditions $y_{0}, y_{0}^{\prime}$. A second-order nonlinear equation

$$
y^{\prime \prime}=R\left(z, y, y^{\prime}\right), \quad z \in \mathbb{C},
$$


where $R$ is rational in $y, y^{\prime}$ and analytic in $z$ (such as the complexification of (1)), is said to have the Painlevé property or to be of Painlevé type if it is free of movable critical points. We now show that (1) is not of Painlevé type (see Ablowitz, Clarkson [1] or Ince [16] for more information on such equations).

Theorem 2. Eq. (1) is not of Painlevé type.

Proof. Clearly (1) is rational in $y, y^{\prime}$ and analytic in $x$. Let $w(x)=1+y(x)$ in order to place the singularity at $w=0$. Then (1) becomes

$$
w^{\prime \prime}=\frac{1}{4} e^{-x}(w-1)+\frac{e^{-2 x}}{w}
$$

which has the form $w^{\prime \prime}=L(x, w)\left(w^{\prime}\right)^{2}+M(x, w) w^{\prime}+N(x, w)$ with $L \equiv 0$ and $M \equiv 0$. According to the analysis of equations of this type given by Ince [16, p. 321 -325] (Sections 14.21 and 14.22), since $L \equiv 0$, the first necessary condition for the absence of movable branch points is satisfied. However, since $N(x, w)$ has a pole at $w=0$, the second necessary condition, which requires that the poles of $M$ and $N$ be included among those of $L$, is violated. Thus, (1) has movable branch points, and is therefore not of Painlevé type.

Theorem 2 is proved.

Remark 2. In addition, the ARS test [2] for the Painlevé property requires that the general solution be expressible in a series about a movable pole $x_{0}$ of order $p$, i.e.,

$$
y=\sum_{n=0}^{\infty} a_{n}\left(x-x_{0}\right)^{n+p}, \quad p \in \mathbb{Z}^{-},
$$

containing two arbitrary constants, one of which is $x_{0}$. Our analysis reveals that the leadingorder term for (1) includes a logarithm, so that the equation admits movable branch points, which is further confirmation that it is not of Painlevé type. The asymptotic behavior of the solutions of (1) near such singularities is given by Theorem 5 below. Finally, our calculations show that (1) does not possess a nontrivial symmetry group, so that reduction or integration of the equation by such methods is also not possible.

Remark 3. We make the convention that a solution with a singularity at $x_{0}$ will be assumed to exist only up to $x_{0}$ and not past it. It follows that any solution of (1) without a singularity is a classical solution on $[0, \infty)$ and it must satisfy either $y(x)<-1$ for all $x \in[0, \infty)$ or $y(x)>-1$ for all $x \in[0, \infty)$ (cf., Remark 1).

Theorem 3. Let $y$ denote a solution of (1) with the property that for some $x_{0} \in(0, \infty), y(x)$ exists on $\left[0, x_{0}\right]$. If $y\left(x_{0}\right)=-1$, then both $\left|y^{\prime}\right|$ and $\left|y^{\prime \prime}\right|$ become infinite at $x_{0}$.

Proof. For a given solution $y$ there is no loss of generality in assuming that the $x_{0}$ in question is the smallest such point. Thus, let $y(x)>-1$ for every $x$ in $\left[0, x_{0}\right)$. Observe that for a given $x_{0}$, a function $f\left(x_{0}\right)$ can be defined by considering only the first, third, and fifth terms of the left-hand side of (5). Its explicit form is

$$
\frac{1}{2} y^{\prime}\left(x_{0}\right)^{2}+e^{-2 x_{0}} \ln \left(1+y\left(x_{0}\right)\right)+2 \int_{0}^{x_{0}} e^{-2 t} \ln (1+y(t)) d t=f\left(x_{0}\right) .
$$


Let $x_{0}>0$ and let $y$ be a solution with initial data set $\left(y_{0}, y_{0}^{\prime}\right)$ such that $y\left(x_{0}\right)=-1$. Then $f$ in (8) can be expressed via (5) as

$$
f\left(x_{0}\right)=\frac{1}{2} y_{0}^{\prime 2}+\frac{1}{8} y_{0}^{2}+\ln \left(1+y_{0}\right)-\frac{1}{8} e^{-x_{0}}-\frac{1}{8} \int_{0}^{x_{0}} e^{-t} y(t)^{2} d t
$$

a quantity that is necessarily finite since $y$ is continuous on $\left[0, x_{0}\right]$, and clearly, $y_{0} \neq-1$.

Now, we estimate the integral appearing in (8). Since $y$ is continuous on $\left[0, x_{0}\right]$ and $y\left(x_{0}\right)=$ $=-1$ there is a $\delta>0$ such that $y(x)<-1 / 2$ for $x \in\left(x_{0}-\delta, x_{0}\right]$, a $\delta$ which we now fix. Hence $\ln (1+y(x))<-\ln 2$ for such $x$. It follows that

$$
\int_{0}^{x_{0}} e^{-2 t} \ln (1+y(t)) d t \leq \int_{0}^{x_{0}-\delta} e^{-2 t} \ln (1+y(t)) d t-\ln 2 \int_{x_{0}-\delta}^{x_{0}} e^{-2 t} d t
$$

so that the integral on the left of (9) is bounded above. Thus, in order for $f\left(x_{0}\right)$ to remain finite, we must have $y^{\prime}\left(x_{0}\right)^{2}=\infty$ in (8). That $\left|y^{\prime \prime}\left(x_{0}\right)\right|$ is also infinite follows immediately from (1).

In the event that $y(x)<-1$ for every $x$ in $\left[0, x_{0}\right)$, we repeat the above argument with the estimate $\ln (-1-y(x))<-\ln 2$ for $x$ chosen so that $y(x)>-3 / 2$. The remaining argument is similar.

Theorem 3 is proved.

The next result complements Theorem 2 above and shows that our equation is actually free of any solutions which become infinite on $(0, \infty)$ (even though it does, in some cases, possess solutions the first and second derivatives of which become infinite).

Theorem 4. Let $y$ be any solution of (1). Then there is no point $x_{0} \in[0, \infty)$ such that $\left|y\left(x_{0}\right)\right|=\infty$.

Proof. If $y(x) \geq 0$ up to such a point $x_{0}$, then its existence contradicts Corollary 2 above at that point. The general situation is similar, although slightly more technical. So assume, on the contrary, that there exists a solution $y$ and $x_{0}>0$ with $\left|y\left(x_{0}\right)\right|=\infty$. There does not exist $x_{1} \in\left(0, x_{0}\right)$ with $y\left(x_{1}\right)=-1$ since, otherwise, the solution would terminate at $x_{1}$ (cf., Remark 3).

Thus, $y \in C\left[0, x_{0}\right)$ and $y \in C[0, b]$ for every $b<x_{0}$. For such a $b<x_{0}$ we use (5) over $[0, b]$ in order to estimate the terms near the singularity $x_{0}$. Doing away with the positive terms we have the estimate

$$
e^{-2 b} \ln |1+y(b)|+2 \int_{0}^{b} e^{-2 t} \ln |1+y(t)| d t \leq I_{0},
$$

where, we recall, $I_{0}$ is defined in (6). Passing to the limit as $b \rightarrow x_{0}^{-}$in (10), using the fact that $\left|y\left(x_{0}\right)\right|=\infty$, and recalling that $I_{0}$ is a constant for a given solution, we obtain

$$
\int_{0}^{x_{0}} e^{-2 t} \ln |1+y(t)| d t=-\infty
$$


Since $\left|y\left(x_{0}\right)\right|=\infty$, there is a $b<x_{0}$ such that $|1+y(t)| \geq 1$ for $t \in\left[b, x_{0}\right)$. Fixing such a $b$ we find that

$$
\begin{aligned}
\int_{0}^{x_{0}} e^{-2 t} \ln |1+y(t)| d t & =\int_{0}^{b} e^{-2 t} \ln |1+y(t)| d t+\int_{b}^{x_{0}} e^{-2 t} \ln |1+y(t)| d t= \\
& =C+\int_{b}^{x_{0}} e^{-2 t} \ln |1+y(t)| d t \geq C,
\end{aligned}
$$

where $C$ is a constant determined by the boundedness of the first integral on the right, since $y(x) \neq-1$ on $[0, b]$. However, this contradicts (11).

Theorem 4 is proved.

Corollary 3. Let $y$ be a solution of (1) and $x_{0}>0$. If $y^{\prime \prime}\left(x_{0}\right)$ is finite, then both $y\left(x_{0}\right)$ and $y^{\prime}\left(x_{0}\right)$ exist and are finite.

Proof. Clearly, $y\left(x_{0}\right)$ is finite by Theorem 4. By the identity (5) employed over the interval $\left[0, x_{0}\right], y^{\prime}$ cannot be infinite since all other quantities therein are finite (as $\left.y\left(x_{0}\right) \neq-1\right)$.

Remark 4. The fundamental existence and uniqueness theorem now guarantees that if a solution $y$ is such that $\left|y^{\prime \prime}\right|<\infty$ on $(0, \infty)$, then y exists on $[0, \infty)$.

A glance at the particular solution of (1) defined by $y_{0}=0, y_{0}^{\prime}=0$ shows that its second derivative becomes unbounded at the value $x_{0} \approx 2.366786778$. Thus, combining Theorems 3 , 4 , we see that the particular solution defined by these zero boundary conditions at $x=0$ is one which is finite, but with first and second derivatives which become infinite. The next result describes the behavior of such solutions near a singularity.

Theorem 5. The asymptotic behavior of a solution y of (1) near a finite singularity $x_{0}>0$ is given by

$$
y(x) \sim-1+\sqrt{2} e^{-x_{0}}\left(x-x_{0}\right)\left[-\ln \left(x-x_{0}\right)\right]^{1 / 2}, \quad x \rightarrow x_{0}^{+},
$$

and

$$
y(x) \sim-1+\sqrt{2} e^{-x_{0}}\left(x_{0}-x\right)\left[-\ln \left(x_{0}-x\right)\right]^{1 / 2}, \quad x \rightarrow x_{0}^{-},
$$

where the positive square root of the logarithmic term is taken if $y>-1$, and the negative if $y<-1$.

Proof. Let $w(x)=\frac{1}{1+y(x)}$ in order that $|w| \rightarrow \infty$ at the singularity, and transform (1) into

$$
w w^{\prime \prime}-2\left(w^{\prime}\right)^{2}-e^{-2 x} w^{4}+\frac{1}{4} e^{-x}\left(w^{3}-w^{2}\right)=0 .
$$

Let $\tau=x-x_{0} \in \mathbb{C}$ and $w=a \tau^{p}$. Then a leading-order balance requires that $p=-1$, but this forces $a=0$, which indicates that a logarithmic term must be present at leading order. Thus, 
put $w=a \tau^{-1}(\log \tau)^{q}$. Then the dominant terms in the equation are

$$
\begin{aligned}
w w^{\prime \prime} & \sim 2 a^{2} \tau^{-4}(\log \tau)^{2 q}-3 a^{2} q \tau^{-4}(\log \tau)^{2 q-1}+O\left(\tau^{-4}(\log \tau)^{2 q-2}\right) \\
\left(w^{\prime}\right)^{2} & \sim a^{2} \tau^{-4}(\log \tau)^{2 q}-2 a^{2} q \tau^{-4}(\log \tau)^{2 q-1}+O\left(\tau^{-4}(\log \tau)^{2 q-2}\right) \\
w^{4} & \sim a^{4} \tau^{-4}(\log \tau)^{4 q}
\end{aligned}
$$

and a balance of these terms requires that $2 q-1=4 q$, i.e., $q=-1 / 2$, from which we obtain $a^{2}=-e^{2 x_{0}} / 2$. Thus,

$$
w \sim \frac{i e^{x_{0}}}{2^{1 / 2}} \tau^{-1}(\log \tau)^{-1 / 2}
$$

and

$$
y=-1+\frac{1}{w} \sim-1-2^{1 / 2} i e^{-x_{0}} \tau(\log \tau)^{1 / 2} .
$$

In order to adapt the above to the real solutions considered herein, let $x_{0} \in \mathbb{R}$ and $\tau=x-x_{0}>$ $>0$. Then

$$
y \sim-1 \pm \sqrt{2} e^{-x_{0}}\left(x-x_{0}\right) \sqrt{-\ln \left(x-x_{0}\right)}, \quad x \rightarrow x_{0}^{+} .
$$

For solutions with $x<x_{0}$, the result is not altered if we let $\tau=x_{0}-x>0$, in which case

$$
y \sim-1 \pm \sqrt{2} e^{-x_{0}}\left(x_{0}-x\right) \sqrt{-\ln \left(x_{0}-x\right)}, \quad x \rightarrow x_{0}^{-} .
$$

Theorem 5 is proved.

By Theorem 5, it is clear that at a finite singularity $x_{0}, y\left(x_{0}\right)=-1$ and that both $y^{\prime}$ and $y^{\prime \prime}$ are infinite at $x_{0}$, in confirmation of Theorems 3,4 above.

2.2. On the non-oscillation of solutions. In this section we assume, unless otherwise specified, that the solutions under consideration have no singularities. As stated in Remark 3, we shall call such solutions classical solutions on $[0, \infty)$.

As is the custom in oscillation theory of differential equations, we say that an equation of the second order (whether linear or not) is oscillatory on a half-line provided that every (non-trivial) solution has arbitrarily large zeros. An equation is said to be non-oscillatory if it has at least one non-oscillatory solution. In addition, a solution is said to be disconjugate on an interval provided that it has at most one zero within it. For more information regarding such solutions in the linear case, the reader may refer to Swanson [27], although we note that a recent comprehensive survey of nonlinear oscillation theory is lacking (cf., Kartsatos [19]). Now, returning to our nonlinear problem (1), according to our convention (see Remark 3), such an oscillating solution will generally belong to the class of solutions such that $y(x)>-1$ for every $x \in[0, \infty)$, unless it is a solution with a critical point.

Lemma 3. Solutions of (1) cannot be identically constant on an interval $\mathcal{I} \subseteq[0, \infty)$.

Proof. Otherwise it is easy to see that its values $y(x)$ must satisfy the quadratic equation $y(x)^{2}+y(x)+4 e^{-x}=0$, in $\mathcal{I}$, by Eq. (1), which is a contradiction.

The next result is actually a special case of another result proved in the next section, but it is of independent interest as its proof can be used in more general situations (see the remark following the lemma). 
Lemma 4. Equation (1) has no non-negative oscillatory solutions. Indeed, every non-negative solution has at most one zero in $[0, \infty)$, and so is disconjugate there.

Proof. We proceed by contradiction. Should $y(x)$ be oscillatory it must have three zeros $x_{1}<x_{2}<x_{3}$. A double application of Rolle's theorem to $y(x)$ on $\left[x_{1}, x_{2}\right],\left[x_{2}, x_{3}\right]$ gives the existence of points $x_{1}^{\prime}<x_{2}^{\prime}$ such that $y^{\prime}\left(x_{i}^{\prime}\right)=0$, for $i=1,2$. This, in turn, yields the existence of another point $x_{1}^{\prime \prime} \in\left[x_{1}^{\prime}, x_{2}^{\prime}\right]$ such that $y^{\prime \prime}\left(x_{1}^{\prime \prime}\right)=0$. Using (1) we see that $y\left(x_{1}^{\prime \prime}\right)$ must be a root of the quadratic equation $y^{2}+y+4 e^{-x_{1}^{\prime \prime}}=0$. However, a glance at its discriminant shows that the quantity $y\left(x_{1}^{\prime \prime}\right)=\left(-1 \pm \sqrt{1-16 e^{-x_{1}^{\prime \prime}}}\right) / 2$ evidently satisfies $-1<y\left(x_{1}^{\prime \prime}\right)<0$, a contradiction. Next, if a non-negative solution has two zeros in $(0, \infty)$, then its derivative must have two zeros as well. Thus, its second derivative must vanish somewhere, which contradicts the non-negativity hypothesis.

Finally, let $y(0)=0$ and $y\left(x_{0}\right)=y^{\prime}\left(x_{0}\right)=0$, where without loss of generality we can assume that $x_{0}$ is the first such zero. Since $y(x)>0$ in a neighborhood of $x_{0}$ (and since $y$ cannot be identically constant there by Lemma 3) it follows that $y^{\prime \prime}\left(x_{0}\right) \geq 0$. However, a direct calculation using (1) shows that $y^{\prime \prime}\left(x_{0}\right)<0$, a contradiction.

Lemma 4 is proved.

Remark 5. Of course, non-negative oscillatory solutions cannot exist for second order linear homogeneous equations but they can, indeed, exist for non-homogenous ones. For example, $y(x)=1-\sin x$ satisfies such an equation and is non-negative and oscillatory. On the other hand, the proof of this lemma is easily modified to show that if $g: \mathbb{R}^{+} \times \mathbb{R} \rightarrow \mathbb{R}$ and $g(x, y)>0$ whenever $y \geq 0$, then the equation $y^{\prime \prime}(x)+g(x, y(x))=0$ has no non-negative oscillatory solutions among those that are continuable to $\mathbb{R}^{+}$.

In the next section we will prove that all solutions with or without singularities on $[0, \infty)$ are, in fact, non-oscillatory and admit at most two zeros.

3. Asymptotic behavior. In this section we characterize the global asymptotic behavior of all the solutions of (1) in terms of their initial data set $\left(y_{0}, y_{0}^{\prime}\right) \in \mathbb{R}^{2}$, using the results of the proof of Theorem 6 above. In so doing we will show the existence of solutions which admit points where their second derivatives (and hence their first, by Theorem 3) become infinite.

Lemma 5. For any set of real initial data $\left(y_{0}, y_{0}^{\prime}\right) \in \mathbb{R}^{2}$ with $y_{0} \neq-1$ we have $y^{\prime \prime}(0) \equiv y_{0}^{\prime \prime} \neq 0$.

Proof. Note that when $y_{0}=-1$ we cannot define a classical solution since its second derivative is automatically undefined. For $y_{0} \neq-1$ we use (1) and see that $y_{0}^{\prime \prime}=0$ if and only if $y_{0}^{2}+y_{0}+4=0$, which is impossible. The result follows.

Next, we characterize the solutions of (1) in terms of their asymptotic behavior, the following series of lemmata providing the proof of Theorem 6 below.

Theorem 6. Equation (1) admits four families of solutions: Specifically, if y is such a solution then either

1) $y(x) \rightarrow+\infty$, as $x \rightarrow \infty$,

2) $y(x) \rightarrow C$, as $x \rightarrow \infty$ where $C$ is a constant, $C \neq-1$,

3) $y(x) \rightarrow-\infty$, as $x \rightarrow \infty$,

4) $y(x)$ has a finite singularity where $y^{\prime \prime}(x)$ becomes infinite, so $y(x)$ cannot be continued past this point.

Terminology: In the following lemmas the reference to the types (1), (2), (3), (4) refer to the types of solutions (1)-(4) respectively in the statement of Theorem 6. 
Lemma 6. Let $y_{0} \geq 0, y_{0}^{\prime}>0$. Then the corresponding solutions are of type (1), (2) or (4).

Proof. Given that $y_{0}>0, y_{0}^{\prime}>0$, then $y_{0}^{\prime \prime}<0$ and so, by continuity, there exists a point $a>0$ such that $y(x)>0, y^{\prime}(x)>0, y^{\prime \prime}(x)<0$ for $x \in[0, a]$. It follows from this that $y$ is increasing and $y^{\prime}$ is decreasing on $[0, a]$. There are now two possibilities:

Either there is a (smallest) point $c>a$ such that $y^{\prime}(c)=0$, or there is no such point, in which case $y^{\prime}(x)>0$ for every $x \in[0, \infty)$. (We note that global existence is obtained in the latter case because of the boundedness of $y$ and so of $y^{\prime}$ on bounded intervals, cf. Remark 4 and Corollary 3 ).

In the latter case, since $y^{\prime}$ is decreasing (it is bounded above) and $y^{\prime}(x)$ has a limit $\beta \geq 0$ as $x \rightarrow \infty$. If $\beta>0$ then it is a simple matter to see that $y(x) \rightarrow+\infty$ as $x \rightarrow \infty$ and so (1) holds. On the other hand, $\beta=0$ is only possible in the case of asymptotically constant solutions $(y(x) \rightarrow C$ for some positive constant $C$, as $x \rightarrow \infty)$ or solutions for which (1) holds. Thus, a solution is either of type (1) or (2) in this special case.

In the former case, let $c>a$ be such that $y^{\prime}(c)=0$, and without loss of generality we can assume that $c$ is the first such point. Since $y(c)>0$ we have $y^{\prime \prime}(c)<0$ so that $c$ is a local maximum (recall that $y(x)$ cannot be identically constant past $c$ on account of Lemma 3 ). Thus, there exists $b>c$ such that $y^{\prime}(x)<0$ for $x \in(c, b]$ and since $y^{\prime}$ is decreasing there it follows that for all $x>b$ such that $y(x) \geq 0$, we must have $y^{\prime}(x)<y^{\prime}(b)<0$. This, however, forces the existence of a point $d>c$ such that $y(d)=0$. Now we employ (1) to find that $y^{\prime \prime}(d)<0$. Because of this there now exists $b_{1}$ such that $y(x)<0, y^{\prime \prime}(x)<0$, and so $y^{\prime}(x)$ is decreasing for $x \in\left(d, b_{1}\right]$. Arguing as before we have $y^{\prime}(x)<y^{\prime}(d)<0$ in a right-neighborhood of $x=d$. However this implies that $y(x) \leq y(d)+y^{\prime}(d)(x-d)$, for all $x$ in this neighborhood. It follows that if this $y$ is continuable to $\mathbb{R}^{+}$, then $y(x) \rightarrow-\infty$ as $x \rightarrow \infty$. But this forces the existence of a point $x_{0}>d$ such that $y\left(x_{0}\right)=-1$ by continuity (since $y_{0}>0$ ). Hence such a solution is of type (4). It follows that for such initial conditions our solutions are either of type (1), (2) or (4) in Theorem 6.

The case where $y_{0}=0$ is similar to the opening paragraph to this proof and so is omitted. There are then the three possibilities again, type (1), (2) or (4), depending on the size of the values of $y_{0}^{\prime}$.

Lemma 6 is proved.

Lemma 7. Let $y_{0}>0, y_{0}^{\prime} \leq 0$. Then the corresponding solutions all have infinite second (and hence first) derivatives at a finite point (i.e., of type (4)).

Proof. We argue as in Lemma 6 above and thus sketch the proof. Consider first the case where $y_{0}>0, y_{0}^{\prime}=0$. Then $y_{0}^{\prime \prime}<0$ so that there exists a point $a>0$ such that $y(x)>0$, $y^{\prime}(x)<0, y^{\prime \prime}(x)<0$ for $x \in[0, a]$. So, $y^{\prime}$ is decreasing on $[0, a]$ and the inequality $y^{\prime}(x)<$ $<y^{\prime}(a)<0$ is in force whenever the derivative is defined. Thus, there is a point $b>a$ such that $y(b)=0$ and so $y^{\prime \prime}(b)<0$, by (1). It follows that $y\left(x_{0}\right)=-1$ for some $x_{0}>b$, i.e., $y$ is of type (4). The proof where $y^{\prime}(0)<0$ is similar and so we omit it.

Lemma 7 is proved.

Lemma 8. Let $-1<y_{0}<0, y_{0}^{\prime}>0$. Then the solutions are either of type (1), (2) or (4).

Proof. Once again we sketch the proof, which in this case parallels that of Lemma 6. For $y_{0}, y_{0}^{\prime}$ as given there exists a point $a>0$ such that $y(x)<0, y^{\prime}(x)>0$, for $x \in[0, a]$. Next, Lemma 5 indicates that $y_{0}^{\prime \prime} \neq 0$, and in fact, it is the case that $y_{0}^{\prime \prime}<0$. This is because the alternative $y_{0}^{\prime \prime}>0$ is equivalent (by (1)) to the inequality $y_{0}\left(1+y_{0}\right)+4<0$ for $-1<y_{0}<0$. 
However, this last quadratic inequality is impossible. Thus, we can restrict $a$ further, if need be, to obtain that $y(x)<0, y^{\prime}(x)>0, y^{\prime \prime}(x)<0$ in $[0, a]$ (so that $y^{\prime}$ is decreasing once again). There are now the two usual possibilities (see the proof of Lemma 6): Either there is a (smallest) point $c>a$ such that $y^{\prime}(c)=0$, or there is no such point $c$ in which case $y^{\prime}(x)>0$ and decreasing on $[0, \infty)$.

Clearly, the existence of such a point $c$ gives a local maximum for $y$. In addition, since $y^{\prime}$ is decreasing to the right of $c$ we must have $y(x)=-1$ for some $x=x_{0}>c$. Hence, the solution is of type (4).

The alternative that $y^{\prime}(x) \neq 0$ gives us $y^{\prime}(x)>0$. Using standard arguments we can now conclude (see above) that there exists a point $b$ at which $y(b)=0$ (and again $y^{\prime \prime}(b)<0$.) If $y^{\prime}(x)$ is bounded away from zero it follows that $y(x) \rightarrow \infty$ as $x \rightarrow \infty$. On the other hand, if $y^{\prime}(x) \rightarrow 0$ as $x \rightarrow \infty$ then either $y$ is of type (1) or type (2).

Lemma 8 is proved.

Lemma 9. Let $-1<y_{0} \leq 0, y_{0}^{\prime} \leq 0$. Then the solutions are of type (4).

Proof. For there is an interval of the form $[0, a]$ in which we have $-1<y(x)<0, y^{\prime}(x)<0$, $y^{\prime \prime}(x)<0$, for $x \in[0, a]$ (see Lemma 8 above). It follows that $y^{\prime}(x)<0$ and decreasing for $x>a$ and so we must have $y(x)=-1$ for some $x=x_{0}>a$. Once again, the solution is of type (4). The case $y_{0}=0$ is similar, since there is a neighborhood $[0, a]$ in which $y(x)<0$, $y^{\prime}(x)<0, y^{\prime \prime}(x)<0$ for $x \in[0, a]$, etc.

Lemma 9 is proved.

Lemma 10. Let $y_{0}<-1$. If $y_{0}^{\prime}>0$, the solution is of type (4). On the other hand, if $y_{0}^{\prime} \leq 0$, the solution is either of type (2), (3) or (4).

Proof. The proof is very similar to that of Lemma 6. For there is an interval of the form $[0, a]$ in which we have either $y(x)<-1, y^{\prime}(x)<0, y^{\prime \prime}(x)>0$, for $x \in[0, a]$ or $y(x)<-1$, $y^{\prime}(x)>0, y^{\prime \prime}(x)>0$ (see Lemma 6 above). In either case it follows that $y^{\prime}(x)$ is monotone for every $x>a$ and so we may have either $y(x)=-1$ for some $x=x_{0}>a, y(x) \rightarrow-\infty$ as $x \rightarrow \infty$ (if $y^{\prime}(x) \rightarrow-\beta<0$ ), or $y(x) \rightarrow-\infty$ or $y(x) \rightarrow C$ as $x \rightarrow \infty$ (if $\left.y^{\prime}(x) \rightarrow 0\right)$, corresponding to the stated initial conditions. Thus, $y$ is either of type (2), (3) or (4).

Lemma 10 is proved.

A consequence of the proofs of these Lemmas is that every solution of (1) has at most one point in $(0, \infty)$ where its derivative can vanish, so one can derive that

Corollary 4. Every solution of (1) has at most two zeros on its interval of existence (i.e., every solution is non-oscillatory).

Referring to Fig. 1 the initial conditions for the solutions reckoned from top to bottom are: $(2,1),(2,0.744448),(2,0.6)$, respectively. The top-most follows an increasing linear trend (type (1) solution) given by the line $y_{0}^{\prime}=0.200043 y_{0}+2.8205$, the middle one is approaching a constant value $C \approx 2.70734$ (type (2) solution), while the bottom one eventually hits a singularity at $x_{0} \approx 32.13$ (type (4) solution).

An example of a type (3) solution is given below in Fig. 2 where the initial conditions are $(-2,-1)$. 


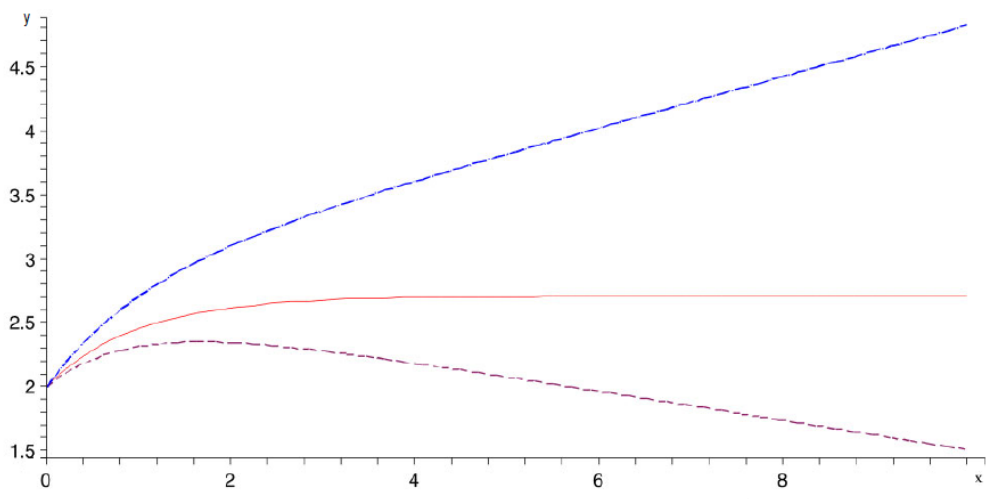

Fig. 1

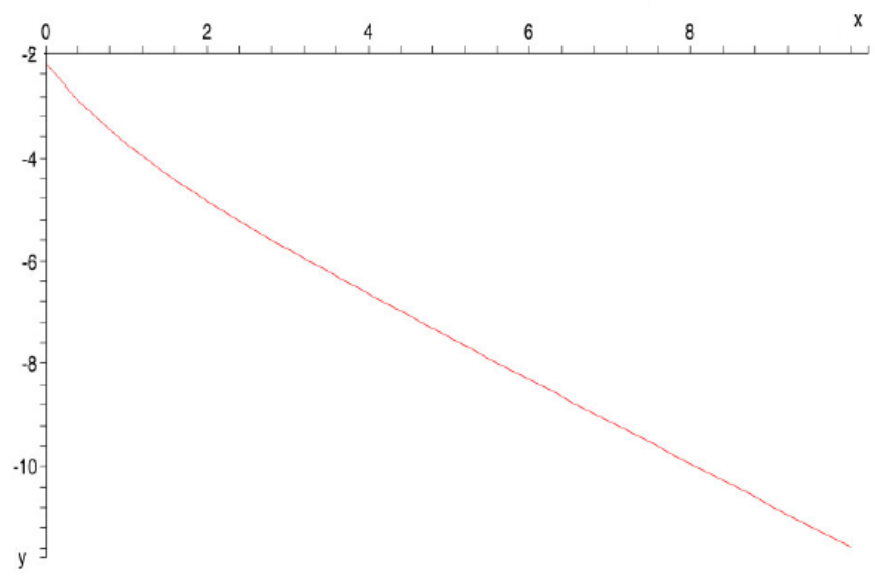

Fig. 2

3.1. Asymptotics. Combining Lemmas $6-10$ in the previous section we can infer that if $y$ is any solution of (1) defined on the whole half-axis then its derivative $y^{\prime}(x) \rightarrow \beta$ where $\beta>0$, $\beta<0$ or $\beta=0$ ( $\beta= \pm \infty$ occurs when we have a finite singularity). Next, we show that the technique presented in [10] can be modified so that in addition to the particular solution appearing in Theorem 1 there is a continuum of asymptotically constant solutions of (1) corresponding to the situation where $y^{\prime}(x) \rightarrow 0$. We recall that $y$ is said to be asymptotically constant as $x \rightarrow \infty$, and write $y(x) \sim M$, if $y(x)=M+o(1)$ as $x \rightarrow \infty$, where $M$ is a constant. As well, we say that a solution is asymptotically linear if it is of the form $y(x)=a x+b+o(1)$ as $x \rightarrow \infty$, for suitable constants $a, b$. We usually write $y(x) \sim a x+b$ to mean that $y$ is asymptotic to the line $a x+b$ as $x \rightarrow \infty$.

We proceed to show that the family of solutions (types (1), (2) and (3)) described in Theorem 6 consists solely of asymptotically linear solutions (not excluding the asymptotically cons- 
tant ones). From this we conclude that either a solution of (1) has a singularity (type (4)) or it is asymptotically linear, thus giving a complete characterization of all the solutions of (1).

Lemma 11. Let $y$ be any solution of (1) that is not of type (4) in Theorem 6. Then

$$
\lim _{t \rightarrow \infty} \frac{y(t)}{t}=\beta
$$

and $y^{\prime}(t)-\beta \in L^{1}(0, \infty)$.

Proof. From the proof of Theorem 6 we know that $y(t)$ exists on $[0, \infty)$ and there is a $\beta \in \mathbb{R}$ such that $y^{\prime}(t) \rightarrow \beta$ as $t \rightarrow \infty$. Without loss of generality we assume that $\beta>0$ (the case $\beta<0$ being similar).

Given $\varepsilon>0$ we choose $T_{0}>0$ so large that $\left|y^{\prime}(t)-\beta\right|<\varepsilon / 2$, for all $t>T_{0}$. Fix such a $T_{0}$. Then

$$
\left|y(t)-y\left(T_{0}\right)-\beta\left(t-T_{0}\right)\right| \leq \int_{T_{0}}^{t}\left|y^{\prime}(\tau)-\beta\right| d \tau<\frac{\varepsilon}{2}\left(t-T_{0}\right),
$$

for all $t>T_{0}$. Hence

$$
\left|\frac{y(t)}{t}-\beta\right| \leq \frac{\varepsilon}{2}\left(1-\frac{T_{0}}{t}\right)+\left|\frac{y\left(T_{0}\right)-\beta T_{0}}{t}\right|<\varepsilon
$$

provided we choose $T_{1}>T_{0}$ so large that $\left|\frac{y\left(T_{0}\right)-\beta T_{0}}{t}\right|<\varepsilon / 2$, for all $t>T_{1}$. We deduce that $y(t) / t \rightarrow \beta$ as $t \rightarrow \infty$.

Note that we can now choose $t$ so large that we have $\beta / 2<|y(t) / t|<3 \beta / 2$, for $t>T_{3}$. If necessary, we can increase $t$ further so that $|1+y(t)|=|1+t(y(t) / t)|>1$, for all $t>T_{4}>T_{3}$. It follows that for every $x>T_{4}$,

$$
\int_{x}^{\infty}|t F(t, y(t))| d t<\int_{x}^{\infty}\left(\frac{1}{4} t^{2} e^{-t}\left|\frac{y(t)}{t}\right|+\frac{t e^{-2 t}}{|1+y(t)|}\right) d t<\int_{x}^{\infty}\left(\frac{3}{8} \beta t^{2} e^{-t}+t e^{-2 t}\right) d t<\infty
$$

where

$$
F(t, y)=\frac{1}{4} e^{-t} y+\frac{e^{-2 t}}{1+y}
$$

Hence the integral on the left is absolutely convergent on $[0, \infty)$.

Keeping in mind that $y^{\prime}(t) \rightarrow \beta$ as $t \rightarrow \infty$, we can now integrate (1) over $[x, \infty)$ and so find that

$$
y^{\prime}(x)=\beta+\int_{x}^{\infty} F(t, y(t)) d t
$$

From this and Fubini's theorem we can derive that for all $x>T_{4}$,

$$
\int_{x}^{\infty}\left|y^{\prime}(t)-\beta\right| d t \leq \int_{x}^{\infty} \int_{t}^{\infty}|F(s, y(s))| d s d t=\int_{x}^{\infty}(t-x)|F(t, y(t))| d t<\int_{0}^{\infty} t|F(t, y(t))| d t<\infty .
$$


The second conclusion follows (the proof of the case $\beta<0$ is similar and so is omitted).

Note that when $\beta=0$ one essential change must occur in the argument. Once again, we derive that $y(t) / t \rightarrow 0$ as $t \rightarrow \infty$. However, we must now choose $T$ so large that $|1+y(t)| \geq$ $\geq \varepsilon>0$ for all $t>T$, which is possible since $|y(x)| \rightarrow \infty$ or $|y(x)| \rightarrow C \neq-1$. Thus, $y^{\prime}(t) \in$ $\in L^{1}(T, \infty)$. In addition, since $y$ is not a type (4) solution, it follows that the values $y(t)$ must be bounded away from $t=-1$, for every value of $t$. So there is a $\delta>0$ such that $|1+y(t)|>\delta$ for all $t \in[0, T]$, from which we get $y^{\prime}(t) \in L^{1}(0, T)$, and this produces the desired integrability over $[0, \infty)$.

Lemma 11 is proved.

Corollary 5. If $y$ is not a type (4) solution of (1) then it is asymptotically linear (or asymptotically constant in the case where $\beta=0)$.

Proof. Since $y^{\prime}-\beta \in L^{1}(0, \infty)$,

$$
y(x)-y(0)-\beta x=\int_{0}^{x}\left(y^{\prime}(t)-\beta\right) d t \rightarrow c \quad \text { as } \quad x \rightarrow \infty
$$

so that

$$
y(x)=\beta x+y(0)+c+\left(\int_{0}^{x}\left(y^{\prime}(t)-\beta\right) d t-c\right)=\beta x+y(0)+c+o(1),
$$

i.e., $y(x) \sim \beta x+\alpha$.

Corollary 5 is proved.

The next four lemmas provide a means for approximating (and proving the existence of) these asymptotically linear (or asymptotically constant) solutions using the contraction mapping principle of $[12$, p. 5]. In so doing we obtain estimates for the sizes of the various solutions involved and initial conditions leading to them (more on this in the next section).

Theorem 7. Let $M, N, M>N \geq 0$ be given numbers such that

$$
(3 M-4 N)(N+1) \geq 1 .
$$

Then there is a unique asymptotically constant solution of (1) such that $N \leq y(x) \leq M$ and $y(x) \rightarrow M$ as $x \rightarrow \infty$.

Proof. We specialize the proof of Theorem 2.1 in [10] to this situation (in conjunction with Example 3.5 in [10, p. 6]). We define the space $X=\{u \in C[0, \infty) \mid N \leq u(t) \leq M, t \geq 0\}$, and note that it is a complete metric space with metric induced by the uniform norm on the half-line. We define

$$
F(t, u(t))=\frac{e^{-t}}{4} u(t)+\frac{e^{-2 t}}{1+u(t)}
$$

and note that for $u \in X$, we have (cf., Eq. (2.1) in [10])

$$
\int_{0}^{\infty} t F(t, u(t)) d t \leq \int_{0}^{\infty}\left(\frac{1}{4} t e^{-t} M+\frac{t e^{-2 t}}{1+N}\right) d t=\frac{1}{4}\left(M+\frac{1}{N+1}\right) \leq M-N
$$


by (13). This shows that the mapping $T$ defined by (cf., Eq. (2.4) in [10])

$$
T u(x)=M-\int_{x}^{\infty}(t-x) F(t, u(t)) d t
$$

maps $X \rightarrow X$. In addition, we choose $k(t)$ so that

$$
k(t)=\frac{1}{4} e^{-t}+\frac{e^{-2 t}}{(1+N)^{2}},
$$

where we have fixed a typographical error in the corresponding expression for (14) with $N=0$, $M=1$ in [10] (Example 3.5). Then

$$
\int_{0}^{\infty} t k(t) d t=\frac{1}{4}\left(1+\frac{1}{(N+1)^{2}}\right)<1
$$

by our assumption on $N$ at the outset, as required for the application of the fixed point theorem. It is now an easy matter to verify that $T$ is a contraction on $X$. Therefore its fixed point corresponds to an asymptotically constant solution of (1).

Theorem 7 is proved.

Setting $N=0$ in this theorem immediately gives the next consequence.

Corollary 6. For any $M \geq 1 / 3$ there is a unique asymptotically constant solution of (1) such that $0 \leq y(x) \leq M$ and $y(x) \rightarrow M$ as $x \rightarrow \infty$.

The preceding corollary extends the application of [10, p. 6] in the case where $M=1$ and $N=0$ to a case of general $M, N$. Now we show that among those solutions with $y^{\prime}(x) \rightarrow$ $\rightarrow \beta$ where $\beta>0$ there is a continuum of unique asymptotically linear ones that can also be calculated to an arbitrary degree of precision using the method of successive approximations (which is built-into the contraction mapping principle).

Theorem 8. Let $\mathcal{D}=\left\{(a, b) \in \mathbb{R}^{2} \mid a>0, b>0,3 a-2 b<0,3 b-2 a \geq 1\right\}$. Then for each $a, b \in \mathcal{D}$ there is a unique solution of (1) such that $0 \leq y(x) \leq a x+b$ and $y(x) \sim a x+b$ as $x \rightarrow \infty$.

Proof. The proof is in the same spirit as that of the aforementioned Theorem 2.1 in [10]. We redefine the space

$$
X=\left\{u \in C[0, \infty) \mid 0 \leq \frac{u(t)}{a t+b} \leq 1, t \geq 0\right\}
$$

and note that once again, for fixed $a, b, X$ is a complete metric space with metric induced by the norm

$$
\|u\|_{X}=\sup _{t \geq 0}\left|\frac{u(t)}{a t+b}\right| .
$$


Define the map $T$ on $X$ by

$$
T u(x)=a x+b-\int_{x}^{\infty}(t-x) F(t, u(t)) d t .
$$

Clearly, for $u \in X$ we have $T u(x) \in C[0, \infty)$. Defining $F$ as in Theorem 7 above, a simple estimation shows that for $u \in X$ we get

$$
\int_{0}^{\infty} t F(t, u(t)) d t \leq \int_{0}^{\infty}\left(\frac{1}{4} t e^{-t}(a t+b)+t e^{-2 t}\right) d t=\frac{1}{4}(1+b)+\frac{1}{2} a \leq b
$$

since $(a, b) \in \mathcal{D}$. Observe that since $3 b-2 a \geq 1$ we have $T u(x) \geq a x+b-b=a x \geq 0$, for all $x \geq 0$ (cf., Eq. (2.6) in [10]). Moreover, $T u(x) \leq a x+b$, since the integral is non-negative on $X$. Thus $T$ is a self-map. We choose $k(t)$ as before (see (14) above). Next we show that $T$ is a contraction on $X$. To this end we note that for $u, v \in X$,

$$
\begin{aligned}
\left|\frac{T u(x)-T v(x)}{a x+b}\right| & \leq \frac{1}{a x+b} \int_{x}^{\infty}(t-x)|a t+b| k(t)\left|\frac{u(t)-v(t)}{a t+b}\right| d t \leq \\
& \leq \frac{1}{b} \sup _{t \geq 0}\left|\frac{u(t)-v(t)}{a t+b}\right| \int_{x}^{\infty}(t-x)(a t+b)\left(\frac{1}{4} e^{-t}+e^{-2 t}\right) d t \leq \\
& \leq \frac{1}{b}\|u-v\|_{X} \int_{0}^{\infty} t(a t+b)\left(\frac{1}{4} e^{-t}+e^{-2 t}\right) d t= \\
& =\frac{1}{b}\left(\frac{3}{4} a+\frac{1}{2} b\right)\|u-v\|_{X} \leq \\
& \leq \kappa\|u-v\|_{X}
\end{aligned}
$$

where $0<\kappa<1$ if and only if $3 a<2 b$. Passing to the supremum on the left it follows that $T$ is a contraction on $X$ since $3 a-2 b<0$ by hypothesis. The existence of a fixed point for $T$ is established, and so is the existence of an asymptotically linear solution of (1).

Theorem 8 is proved.

Remark 6. The point $(a, b)=(0,1 / 3)$ in on the boundary of $\mathcal{D}$ and this gives an asymptotically constant solution as we have seen in Theorem 7. Indeed, all the solutions obtained via Theorem 7 appear as boundary points of $\mathcal{D}$. As an application of Theorem 8 we conclude that there is a solution of (1) such that $y(x) \sim x+2$ as $x \rightarrow \infty$ since the point $(1,2) \in \mathcal{D}$.

A result corresponding to Theorem 7 but for negative asymptotically constant solutions is next.

Theorem 9. Let $\alpha>1+1 / \sqrt{3}$, and choose $M \geq \max \{8 / 3, \alpha\}$ so large that

$$
\alpha \leq \frac{1}{2}+\frac{3}{8} M+\frac{1}{8} \sqrt{3} \sqrt{M(3 M-8)} .
$$


Then (1) has a unique asymptotically constant solution y such that

$$
-M \leq y(x) \leq-\alpha, \quad x \geq 0
$$

and $y(x) \sim-M$ as $x \rightarrow \infty$.

Proof. Given $\alpha$ we fix a value of $M$ as per the assumptions. Then the space

$$
X=\{u \in C[0, \infty) \mid-M \leq u(t) \leq-\alpha, t \geq 0\}
$$

is a complete metric space with metric $d$ defined by $d(u, v)=\max _{t \geq 0}|u(t)-v(t)|$. We define a map $T$ on $X$ as usual by setting

$$
T u(x)=-M-\int_{x}^{\infty}(t-x) F(t, u(t)) d t,
$$

where $F$ is defined as before by

$$
F(t, u(t))=\frac{1}{4} u(t) e^{-t}+\frac{e^{-2 t}}{1+u(t)}, \quad u \in X
$$

Note that since $u \in X$ we have $F(t, u(t))<0$ for every $t \geq 0$. In addition, $T u(x)$ is continuous for $x \geq 0$ and $T u(x)+M \geq 0$, for every $x \geq 0$. We now need to estimate $T$ from above in order to show that $T$ is a self-map. Since $u \in X$,

$$
\begin{aligned}
T u(x) & =-M-\int_{x}^{\infty}(t-x)\left(\frac{1}{4} u(t) e^{-t}+\frac{e^{-2 t}}{1+u(t)}\right) d t \leq \\
& \leq-M+\int_{x}^{\infty}(t-x)\left(\frac{1}{4} M e^{-t}+\frac{e^{-2 t}}{\alpha-1}\right) d t \leq \\
& \leq-M+\int_{0}^{\infty}\left(\frac{1}{4} M t e^{-t}+\frac{t e^{-2 t}}{\alpha-1}\right) d t= \\
& =-\frac{3}{4} M+\frac{1}{4(\alpha-1)} .
\end{aligned}
$$

Thus, $T u(x) \leq-\alpha$ provided we can make $-\frac{3}{4} M+\frac{1}{4(\alpha-1)} \leq-\alpha$, an expression that leads to the quadratic inequality $4 \alpha^{2}-(4+3 M) \alpha+3 M+1 \leq 0$. The discriminant being given by $3 M(3 M-8)$, we see that it is necessary that $M \geq 8 / 3$. Furthermore, $M \geq \alpha$ by definition of the set $X$ and so $M \geq \max \{8 / 3, \alpha\}$ as required. A solution of the quadratic inequality is given by choosing $M$ larger still, if necessary, so that $\alpha \leq \frac{1}{2}+\frac{3}{8} M+\frac{1}{8} \sqrt{3} \sqrt{M(3 M-8)}$. Fixing such a value of $M$ we have $T u(x) \leq-\alpha$ and so $T u(x) \in X$. Hence $T: X \rightarrow X$. 
In order to show that $T$ is a contraction on $X$ (and hence continuous) we proceed as follows: For $u, v \in X$,

$$
\begin{aligned}
|T u(x)-T v(x)| & \leq \int_{x}^{\infty}(t-x)|F(t, u(t))-F(t, v(t))| d t \leq \\
& \leq \int_{x}^{\infty}(t-x)\left(\frac{1}{4} e^{-t}|u(t)-v(t)|+\frac{e^{-2 t}|u(t)-v(t)|}{|1+u(t)||1+v(t)|}\right) d t \leq \\
& \leq \int_{x}^{\infty}(t-x)\left(\frac{1}{4} e^{-t}|u(t)-v(t)|+\frac{e^{-2 t}|u(t)-v(t)|}{(\alpha-1)^{2}}\right) d t \leq \\
& \leq d(u, v) \int_{0}^{\infty}\left(\frac{1}{4} t e^{-t}+\frac{t e^{-2 t}}{(\alpha-1)^{2}}\right) d t \leq \\
& \leq \kappa d(u, v),
\end{aligned}
$$

where $\kappa=\left(1+(\alpha-1)^{-2}\right) / 4<1$ since $\alpha>1+1 / \sqrt{3}$ by hypothesis. As a result we find $d(T u, T v) \leq \kappa d(u, v)$ for $u, v \in X$. So $T$ is a contraction and thus has a fixed point $u \in X$. This fixed point corresponds to a solution $y$ with the stated properties.

Theorem 9 is proved.

Remark 7. These methods give rough estimates to the initial conditions that generate the required solution. For example, it follows from Theorem 9 that the solution $y(x)$ with $y(x) \sim$ $\sim-M$ satisfies $-M \leq y_{0} \leq-\alpha$. Thus, in practice it is best to find the smallest interval of length $M-\alpha$.

The final result in this section is an analog of Theorem 8 for negative solutions satisfying the condition $y^{\prime}(x) \rightarrow-\beta$ as $x \rightarrow \infty$ where $\beta>0$. It is somewhat more technical than the stated result since we must stay away from a point where $y=-1$. Its proof is a combination of the proofs of both Theorems 8,9 .

Theorem 10. Let $\alpha>1, a>0, b>0$, and

$$
\mathcal{D}=\left\{(a, b) \in \mathbb{R}^{2} \mid 3 b-2 a \geq 4 \alpha+\frac{1}{\alpha-1}, \frac{3 a-2 b}{3 b-2 a}<\alpha(\alpha-2)\right\}
$$

Then for each $a, b \in \mathcal{D}$ there is a unique solution of (1) such that $-a x-b \leq y(x) \leq-\alpha$ for $x \geq 0$, and $y(x) \sim-a x-b$ as $x \rightarrow \infty$.

Proof. We define a complete metric space by $X=\{u \in C[0, \infty) \mid-a t-b \leq u(t) \leq$ $\leq-\alpha, t \geq 0\}$, with metric $d(u, v)$ defined by the norm

$$
d(u, v)=\sup _{t \geq 0}\left|\frac{u(t)-v(t)}{-a t-b}\right|=\|u-v\|_{X}
$$


As is usual we define the map $T$ on $X$ by

$$
T u(x)=-a x-b-\int_{x}^{\infty}(t-x) F(t, u(t)) d t .
$$

Once again, for $u \in X$ we have $T u(x) \in C[0, \infty)$. First we show that $T$ is a self-map.

To this end, let $u \in X$. Since $u(t)<-\alpha<0$ and $\alpha>1, F(t, u(t))<0$ for every $t \geq 0$. Hence $T u(x)+a x+b \geq 0$, for all $x \geq 0$. In addition, for $u \in X$,

$$
\begin{aligned}
T u(x) & =-a x-b-\int_{x}^{\infty}(t-x) F(t, u(t)) d t= \\
& =-a x-b+\int_{x}^{\infty}(t-x)|F(t, u(t))| d t \leq \\
& \leq-a x-b+\int_{x}^{\infty}(t-x)\left(\frac{1}{4} e^{-t}(a t+b)+\frac{e^{-2 t}}{\alpha-1}\right) d t \leq \\
& \leq-a x-b+\int_{0}^{\infty} t\left(\frac{1}{4} e^{-t}(a t+b)+\frac{e^{-2 t}}{\alpha-1}\right) d t \leq \\
& \leq-a x-b+\frac{1}{4(\alpha-1)}+\frac{1}{2} a+\frac{1}{4} b \leq \frac{1}{4(\alpha-1)}+\frac{1}{2} a-\frac{3}{4} b
\end{aligned}
$$

for each $x \geq 0$. Since $(a, b) \in \mathcal{D}, T u(x) \leq-\alpha$ and so $T: X \rightarrow X$. We show that $T$ is a contraction on $X$. Arguing as in the proof of Theorem 8 we find for $u, v \in X$,

$$
\begin{aligned}
\left|\frac{T u(x)-T v(x)}{-a x-b}\right| & \leq \frac{1}{a x+b} \int_{x}^{\infty}(t-x)|a t+b|\left(\frac{1}{4} e^{-t}+\frac{e^{-2 t}}{(\alpha-1)^{2}}\right)\left|\frac{u(t)-v(t)}{a t+b}\right| d t \leq \\
& \leq \frac{1}{b} \sup _{t \geq 0}\left|\frac{u(t)-v(t)}{a t+b}\right| \int_{x}^{\infty}(t-x)(a t+b)\left(\frac{1}{4} e^{-t}+\frac{e^{-2 t}}{(\alpha-1)^{2}}\right) d t \leq \\
& \leq \frac{1}{b} d(u, v) \int_{0}^{\infty} t(a t+b)\left(\frac{1}{4} e^{-t}+\frac{e^{-2 t}}{(\alpha-1)^{2}}\right) d t,
\end{aligned}
$$

so that

$$
d(T u, T v) \leq \kappa d(u, v)
$$

where the contraction constant

$$
\kappa=\frac{(2 a+b) \alpha^{2}-(4 a+2 b) \alpha+(3 a+2 b)}{4 b(\alpha-1)^{2}}<1
$$


if and only if

$$
(2 a-3 b) \alpha^{2}-(4 a-6 b) \alpha+(3 a-2 b)<0,
$$

a quadratic with discriminant $4\left(3 b^{2}+a b-2 a^{2}\right)=4(b+a)(3 b-2 a)>0$ because $(a, b) \in \mathcal{D}$. In addition, $2 a-3 b<0$, so (15) is equivalent to

$$
\alpha(\alpha-2)>\frac{3 a-2 b}{3 b-2 a}
$$

Thus, with $a, b$ suitably restricted as in Eq. (16), we get $\kappa<1$ and so $T$ is a contraction on $X$. Note that if $\alpha>2$ and $3 a=2 b$, then the first of the two conditions on $a, b$ in $\mathcal{D}$ is sufficient to guarantee the existence of asymptotically linear solutions of this type.

Theorem 10 is proved.

Remark 8. We emphasize once again that one advantage in using fixed point methods to prove the existence of asymptotically linear solutions of (1) lies in the fact that the BanachCacciopoli fixed point theorem actually implies that the method of "successive approximations"(or Picard iterations as they are sometimes called) can be used to approximate the solutions themselves, i.e., the method lends itself to computability (see [15], Chapter 2). Although the Schauder fixed point theorem is more general and can be used here, it only gives existence (without uniqueness) and little or no computable features for the solutions themselves.

Example 1. In order to determine the initial conditions that generate the solution in Theorem 1 , we simply write

$$
y_{n+1}(x)=1-\int_{x}^{\infty}(t-x) F\left(t, y_{n}(t)\right) d t, \quad n \geq 1
$$

where $0 \leq y_{1}(t) \leq 1$ is given by say, $y_{1}(t)=1 / 2$. These iterations must converge to the required solution by the stated fixed point theorem.

Finding the first few iterates and substituting the value $x=0$ in both $y_{n}$ and its derivative gives the approximate values $y_{0}=0.6236, y_{0}^{\prime}=0.49148$ (see Fig. 3 ). The more iterations one uses the better the approximation. Of course, any seed (within the required limits) can be used as a starting value and the iterations will all converge to the unique solution sought.

4. Connecting solutions to initial conditions. In this final section we give information on those asymptotic solutions having prescribed initial conditions and conversely. In so doing we will be in a position to describe the asymptotic behavior of all solutions in a plane of initial 


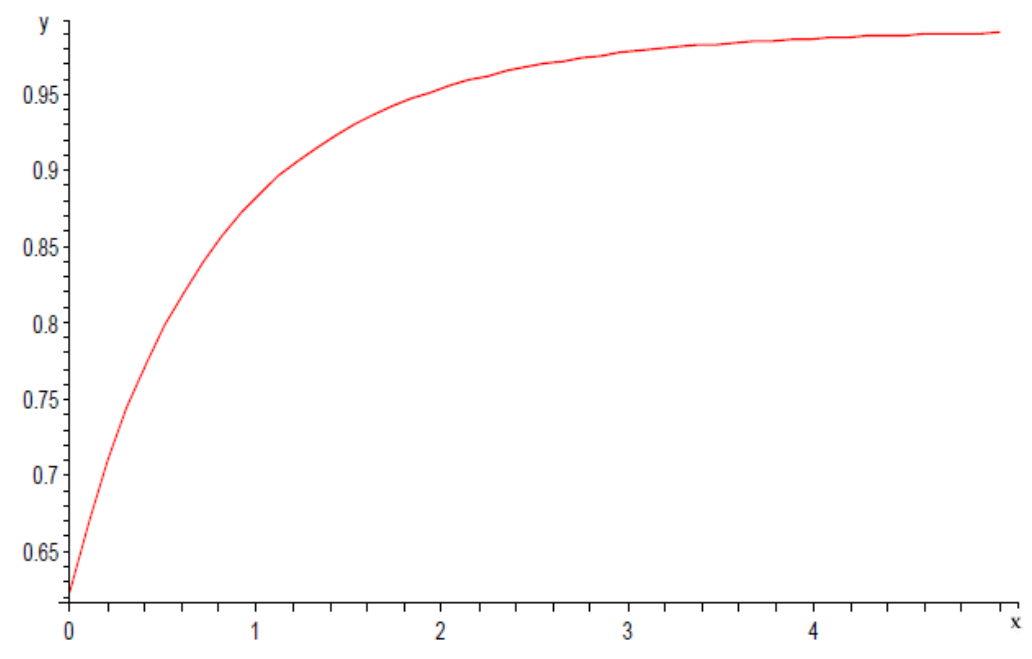

Fig. 3

conditions (see below). It is best to refer to Theorem 6 in the sequel. We recall that $y_{0} \equiv y(0)$, $y_{0}^{\prime} \equiv y^{\prime}(0)$ where all the initial conditions are assumed real. In addition, $I \equiv I_{0}$ is defined as in (6), that is,

$$
I=\frac{1}{2} y_{0}^{\prime 2}+\frac{1}{8} y_{0}^{2}+\ln \left|1+y_{0}\right|
$$

The following result gives a sufficient condition for the existence of solutions with finite singularities on $[0, \infty)$.

Lemma 12. Let $I \leq 0$. Then the corresponding solution of (1) has a point where its second derivative is infinite.

Proof. First we note that the region defined by (17) subject to $I \leq 0$ is contained in the strip $-1<y_{0} \leq 0$, of the $\left(y_{0}, y_{0}^{\prime}\right)$-plane. If $y_{0}^{\prime} \leq 0$ then, by Lemma 9 , the solution has a singularity as stated.

On the other hand, if $y_{0}^{\prime}>0$ then $I<0$. Now, use of Lemma 2 shows that any corresponding solution of (1) must be negative on every interval to the right of $x=0$ in which it is defined. Hence by combining Lemma 8 and Theorem 6 we deduce that $y$ cannot be of type (1) or (2) (since then the solution must be positive somewhere to the right of zero and this contradicts the stipulated negativity above).

Lemma 12 is proved.

We have seen in Theorem 8, Theorem 10 and Corollary 5 that asymptotically linear solutions exist and, in fact, prevail when we assume that the solution is not of type (4). In this spirit, we provide some theoretical (and so exact) relationships between the solution asymptotics and the corresponding initial conditions.

Lemma 13. Let $y$ be a solution of (1) that is asymptotically linear, viz., for some $a, b \in \mathbb{R}$, $y(x) \sim a x+b$ as $x \rightarrow \infty$, where $a=0$ is allowed. Then 


$$
y_{0}=b-\int_{0}^{\infty} t F(t, y(t)) d t
$$

and

$$
y_{0}^{\prime}=a+\int_{0}^{\infty} F(t, y(t)) d t
$$

Conversely, if $\left(y_{0}, y_{0}^{\prime}\right)$ are initial conditions leading to an asymptotically linear solution $y(x)$, then the corresponding constants $a, b$ are given above.

Proof. A double integration of (1) along with Fubini's Theorem gives, for any $x \geq 0$,

$$
y(x)=y_{0}+y_{0}^{\prime} x-\int_{0}^{x}(x-t) F(t, y(t)) d t .
$$

On the other hand, since this same solution is asymptotically linear we have $y(x) \rightarrow a x+b$ and $y^{\prime}(x) \rightarrow a$ as $x \rightarrow \infty$. Hence, by the results in the previous section $y(x)$ also satisfies the integral equation

$$
y(x)=b+a x-\int_{x}^{\infty}(t-x) F(t, y(t)) d t, \quad x \geq 0 .
$$

Since these last two equations must be identical we can differentiate them and thereby find (19). To get (18) we set $x=0$ in both of them. The converse is clear.

Lemma 13 is proved.

Notation. Next, let $\mathcal{S}_{0}$ be the subset of all initial values $y_{0}$ where $-1<y_{0}<\infty$, such that there exists a value of $y_{0}^{\prime}>0$ whose corresponding solution is asymptotically constant.

We undertake a study of this set $\mathcal{S}_{0}$ in the sequel. A combination of Lemma 7, Lemma 8 and Theorem 7 in that order indicates that $\mathcal{S}_{0} \neq \phi$. As a result, those initial values with $y_{0}>-1$ (resp. $y_{0}<-1$ ) leading to asymptotically constant solutions of (1) lie in the strip $y_{0}>-1$, $y_{0}^{\prime}>0$ (resp. $\left.y_{0}<-1, y_{0}^{\prime}<0\right)$ of the $\left(y_{0}, y_{0}^{\prime}\right)$-plane.

It is interesting to note that if $y_{0}=-1$ is a limit point of $\mathcal{S}_{0}$ then $y_{0}^{\prime} \rightarrow+\infty$. That is, the closer we are to the singularity the higher we have to shoot in order to get asymptotically constant solutions (otherwise the solutions "fall back" and become solutions having points where the second derivative is infinite). This is the content of the next result.

Lemma 14. Let $y_{0}^{n} \in \mathcal{S}_{0}$ be a sequence of initial values with $y_{0}^{n} \rightarrow-1$ as $n \rightarrow \infty$. Then the corresponding values $y_{0}^{\prime n} \rightarrow+\infty$ as $n \rightarrow \infty$.

Proof. Let $\left(y_{0}^{n}, y_{0}^{\prime n}\right)$ be such an initial condition and $y_{n}(x)$ be the corresponding solution. Refer to (5) with $y$ replaced by $y_{n}$ and $a=0$. Note that since $y(x)$ is asymptotically constant and $y^{\prime}(x) \rightarrow 0$, as $x \rightarrow \infty$, the left-hand side of (5) is finite. However, the right-hand side of (5) is unbounded unless $\left(y_{0}^{\prime n}\right)^{2}=+\infty$. The result follows. 
Lemma 15. Let $y(x)$ be any asymptotically linear solution of (1). Then $y \in C^{\infty}(0, \infty)$ and there is an expansion of $y$ into a series of initial conditions $y(x)=\sum_{n=0}^{\infty} c_{n}\left(y_{0}, y_{0}^{\prime}\right) x^{n}$ where the coefficients are functions of $y_{0}, y_{0}^{\prime}$ alone and the expansion is absolutely and uniformly convergent for $x$ in compact subsets of $[0, \infty)$.

Proof. Since $F$ is analytic for $t \in \mathbb{C}$ and also for $y \in \mathbb{C} \backslash\{-1\}$ (the complex plane punctured at $y=-1$ ), it follows from standard theorems (cf., [15], Theorem 2.5.2) that every asymptotically linear solution of (1) defined by initial conditions $y_{0}, y_{0}^{\prime}$ where $y_{0} \neq-1$ is necessarily complex analytic and so infinitely differentiable for all $x>0$. In addition,

$$
y(x)=\sum_{n=0}^{\infty} c_{n}\left(y_{0}, y_{0}^{\prime}\right) x^{n}
$$

where the power series converges absolutely and uniformly for $x$ in compact subsets of $[0, \infty)$, and the coefficients are functions of the initial conditions $\left(y_{0}, y_{0}^{\prime}\right)$. The coefficients are easily found by appealing to the differential equation (1) and differentiating as required since $y \in$ $C^{\infty}(0, \infty)$. Indeed, its first few terms are given by $c_{n} \equiv c_{n}\left(y_{0}, y_{0}^{\prime}\right)$.

$$
\begin{aligned}
& c_{0}=y_{0} \\
& c_{1}=y_{0}^{\prime} \\
& c_{2}=-\frac{1}{8}\left(y_{0}+\frac{4}{1+y_{0}}\right) \\
& c_{3}=\frac{1}{24}\left(y_{0}+\frac{8}{1+y_{0}}+y_{0}^{\prime} \frac{\left(3+y_{0}\right)\left(1-y_{0}\right)}{\left(1+y_{0}\right)^{2}}\right) \\
& c_{4}=-\frac{y_{0}\left(127+73 y_{0}^{2}+9 y_{0}^{2}+3 y_{0}^{3}\right)+y_{0}^{\prime}\left(56+40 y_{0}-24 y_{0}^{2}-8 y_{0}^{3}\right)+76+32 y_{0}^{\prime 2}}{134\left(1+y_{0}\right)^{3}}
\end{aligned}
$$

Lemma 15 is proved.

Remark. Eq. (20) can be used to determine the short-term qualitative behavior of any solution of (1). For example, it is easy to see that if $y$ is any solution of (1) with initial conditions $\left(y_{0}, y_{0}^{\prime}\right)$, then for all sufficiently small $x$ for which the solution is defined,

$$
\operatorname{sgn} y^{\prime \prime}(x)=-\operatorname{sgn}\left(1+y_{0}\right)
$$

Thus, we can easily derive from this that the graph of $y$ is concave up (resp. down) whenever $y_{0}<-1$ (resp. $y_{0}>-1$ ) if $x$ is small enough.

In order to gain an understanding of the initial conditions leading to asymptotically constant solutions we require an estimate of the approach of such a solution to its limit. This growth is provided by the next result. 
Lemma 16. Let $y$ be an asymptotically constant solution of (1) with $y(x) \sim M$, where $M \neq 0$, as $x \rightarrow \infty$. Then, for all sufficiently large $x$,

$$
\begin{array}{r}
|y(x)-M|=O\left(e^{-x}\right), \\
\left|y^{\prime}(x)\right|=O\left(e^{-x}\right) .
\end{array}
$$

Proof. First, we use an induction argument to show that if $y_{n}(x), n \geq 1$, denote the successive approximations of $y(x)$, then $F\left(t, y_{n}(t)\right)=O\left(e^{-t}\right)$ as $t \rightarrow \infty$. Setting $y_{1}(t)=M$, for every $t \geq 0$, it is easy to see that $F\left(t, y_{1}(t)\right)=O\left(e^{-t}\right)$ for large $t$, so the statement is true for $n=1$. Assume that $F\left(t, y_{i}(t)\right)=O\left(e^{-t}\right)$, for large $t$, holds for $i=2,3, \ldots, n$. Since

$$
y_{n+1}(x)=M-\int_{x}^{\infty}(t-x) F\left(t, y_{n}(t)\right) d t, \quad n \geq 1
$$

necessarily for every $x \geq 0$, we see that

$$
\left|y_{n+1}(x)-M\right| \leq \int_{x}^{\infty}(t-x)\left|F\left(t, y_{n}(t)\right)\right| d t \leq A \int_{x}^{\infty}(t-x) e^{-t} d t=O\left(e^{-x}\right),
$$

for all sufficiently large $x$, where $A$ is some positive constant (which can be made independent of $n$ ) whose value is immaterial. Passing to the limit we find (22).

On the other hand, since $y^{\prime}(x) \rightarrow 0$ as $x \rightarrow \infty$ we also have (cf., see the proof of Lemma 13 above),

$$
y_{n+1}^{\prime}(x)=\int_{x}^{\infty} F\left(t, y_{n}(t)\right) d t, \quad n \geq 1
$$

Thus, (23) follows from the estimates on $F$ from the first part upon passage to the limit.

Lemma 16 is proved.

Next we show that corresponding to any initial value $y_{0}>-1$ there is an asymptotically constant solution for some appropriate choice of $y_{0}^{\prime}>0$.

Theorem 11. For every $\delta>0$ and $\delta<1 / 2$, there is an asymptotically constant solution $y$ of (1) such that $y(x) \sim-1+2 \delta$ as $x \rightarrow \infty$. For such a solution, its initial values $y_{0}, y_{0}^{\prime}$ satisfy $-1<y_{0}<-1+2 \delta$ and $y_{0}^{\prime}>0$.

Proof. Write $M=-1+2 \delta$ and consider the successive approximations (24)

$$
\begin{aligned}
y_{n+1}(x) & =M-\int_{x}^{\infty}(t-x) F\left(t, y_{n}(t)\right) d t= \\
& =M-\int_{x}^{\infty}(t-x)\left(\frac{1}{4} e^{-t} y_{n}(t)+\frac{e^{-2 t}}{1+y_{n}(t)}\right) d t, \quad n \geq 1 .
\end{aligned}
$$


Choose $X_{0}$ so large that

$$
\frac{1}{4}(3 \delta-1) e^{-x}+\frac{1}{4 \delta} e^{-2 x}<\delta
$$

and

$$
\frac{1}{4}(1-\delta) e^{-x}-\frac{1}{12 \delta} e^{-2 x}<\delta \text { for all } x>X_{0}
$$

and

$$
\alpha \equiv \frac{1}{4 \delta^{2}}\left(e^{-2 X_{0}}+\delta^{2} e^{-X_{0}}\right)<1
$$

Define $y_{0}(x) \equiv-1+\delta$, for every $x \geq 0$. We now show that for $n=0,1,2, \ldots$, we have

$$
-1+\delta \leq y_{n}(x) \leq-1+3 \delta
$$

for all $x>X_{0}$. The result is clear for $n=0$. Assuming (29) true for $n=m$, we estimate $y_{m+1}$ to find

$$
\begin{aligned}
\int_{x}^{\infty}(t-x)\left(\frac{1}{4} e^{-t}(\delta-1)+\frac{e^{-2 t}}{3 \delta}\right) d t & \leq \int_{x}^{\infty}(t-x)\left(\frac{1}{4} e^{-t} y_{m}(t)+\frac{e^{-2 t}}{1+y_{m}(t)}\right) d t \leq \\
& \leq \int_{x}^{\infty}(t-x)\left(\frac{1}{4} e^{-t}(3 \delta-1)+\frac{e^{-2 t}}{\delta}\right) d t
\end{aligned}
$$

which gives

$$
\frac{1}{4}(\delta-1) e^{-x}+\frac{e^{-2 x}}{12 \delta} \leq \int_{x}^{\infty}(t-x) F\left(t, y_{m}(t)\right) d t \leq \frac{1}{4}(3 \delta-1) e^{-x}+\frac{e^{-2 x}}{4 \delta}
$$

from which it follows that

$$
2 \delta-1-\frac{1}{4}(3 \delta-1) e^{-x}-\frac{e^{-2 x}}{4 \delta} \leq y_{m+1}(x) \leq 2 \delta-1-\frac{1}{4}(\delta-1) e^{-x}-\frac{e^{-2 x}}{12 \delta}
$$

for every $x>X_{0}$, and the result follows on account of (27). Thus, (29) is true for every $n \geq 0$ and $x>X_{0}$.

Next, we estimate successive differences in the approximations. Observe that for $m \geq 0$,

$$
\begin{aligned}
y_{m+2}(x)-y_{m+1}(x) & =\int_{x}^{\infty}(t-x)\left(F\left(t, y_{m}(t)\right)-F\left(t, y_{m+1}(t)\right) d t=\right. \\
& =\int_{x}^{\infty}(t-x)\left(\frac{1}{4} e^{-t}\left(y_{m}(t)-y_{m+1}(t)\right)+\frac{e^{-2 t}\left(y_{m+1}(t)-y_{m}(t)\right)}{\left(1+y_{m}(t)\right)\left(1+y_{m+1}(t)\right.}\right) d t .
\end{aligned}
$$


Consequently,

$$
\begin{aligned}
\left|y_{m+2}(x)-y_{m+1}(x)\right| \leq & \sup _{t>x}\left|y_{m+1}(t)-y_{m}(t)\right| \times \\
& \times \int_{x}^{\infty}(t-x)\left(\frac{1}{4} e^{-t}+\frac{e^{-2 t}}{\left(1+y_{m}(t)\right)\left(1+y_{m+1}(t)\right)}\right) d t .
\end{aligned}
$$

However, from (29) we have $\left(1+y_{m}(t)\right)\left(1+y_{m+1}(t)\right) \geq \delta^{2}$ for every $x>X_{0}$. Using this in (30), we get the estimate

$$
\begin{aligned}
\left|y_{m+2}(x)-y_{m+1}(x)\right| & \leq \sup _{t>x}\left|y_{m+1}(t)-y_{m}(t)\right| \int_{x}^{\infty}(t-x)\left(\frac{1}{4} e^{-t}+\frac{e^{-2 t}}{\delta^{2}}\right) d t= \\
& =\sup _{t>x}\left|y_{m+1}(t)-y_{m}(t)\right| \frac{1}{4 \delta^{2}}\left(e^{-2 x}+\delta^{2} e^{-x}\right) \leq \\
& \leq \alpha \sup _{t>x}\left|y_{m+1}(t)-y_{m}(t)\right|, \quad m \geq 0,
\end{aligned}
$$

where $0<\alpha<1$, provided that $x>X_{0}$, by (28). Taking the supremum of both sides of (31), we see that this establishes the convergence of the successive approximations, at least for $x>X_{0}$. The continuity of the limiting function $y(x)$ is also easily proved using these estimates.

Finally, by Lemma 7 and Lemma 9, we must have that for such a solution, $y_{0}^{\prime}>0$. If $y_{0} \geq 0$, then the proof of Lemma 6 shows that $M>0$, which is false. Thus, $-1<y_{0}<0$. Arguing as in the proof of Lemma 8, the solution must satisfy $y(x)<0, y^{\prime}(x)>0$ and $y^{\prime \prime}(x)<0$. But $y^{\prime}(x)$ has a limit at infinity, and if this limit is positive, then we obtain an asymptotically linear solution, which is impossible. Thus, this limit is zero. However, note that $y^{\prime}(x)>0$ throughout its domain, so that $y(x)$ is increasing to its limit. Thus, $-1<y_{0}<-1+2 \delta$.

Theorem 11 is proved.

Remark 10. Combining Theorem 7 and Theorem 11, we obtain that for every initial value $y_{0}>-1$, there is a value of $y_{0}^{\prime}>0$ such that the corresponding solution is asymptotically constant at infinity. A similar result holds for the initial conditions $y_{0}<-1, y_{0}^{\prime}<0$ and is left to the reader.

We show presently that the collection of points $\left(y_{0}, y_{0}^{\prime}\right)$ in $\Pi$ which lead to an asymptotically constant solution is, in fact, a piecewise differentiable curve whose graph we can approximate.

Theorem 12. The set $\mathcal{T}_{0}=\left\{\left(y_{0}, y_{0}^{\prime}\right) \mid y_{0} \in \mathcal{S}_{0}\right\}$ is the graph of a differentiable function on $\mathbb{R} \backslash\{-1\}$.

Proof. Write $y\left(x, y_{0}, y_{0}^{\prime}\right)$ for an asymptotically constant solution of (1) whose initial values are defined by $y(0)=y_{0}, y^{\prime}(0)=y_{0}^{\prime}$. We take it that $y(x) \sim M$, where $M>0$, and consider the relation $y\left(x, y_{0}, y_{0}^{\prime}\right)-M=0$ (or $\left.y^{\prime}\left(x, y_{0}, y_{0}^{\prime}\right)=0\right)$, the idea here being an attempt to approximate this solution. Standard continuous dependence on the initial conditions estimates (e.g., $\left[15\right.$, p. 78]) give that $y\left(x, y_{0}, y_{0}^{\prime}\right)$ is a differentiable function of $\left(y_{0}, y_{0}^{\prime}\right)$. Fixing $x$ and $y_{0}$ for 
the time being, we deduce that $y$ is a differentiable function of $y_{0}^{\prime}$. Since

$$
y\left(x, y_{0}, y_{0}^{\prime}\right)=y_{0}+y_{0}^{\prime} x-\int_{0}^{x}(x-t) F\left(t, y\left(t, y_{0}, y_{0}^{\prime}\right)\right) d t
$$

differentiating both sides with respect to $y_{0}^{\prime}$ we find

$$
\frac{\partial y}{\partial y_{0}^{\prime}}\left(x, y_{0}, y_{0}^{\prime}\right)=x-\int_{0}^{x}(x-t)\left(\frac{1}{4} e^{-t}-\frac{e^{-2 t}}{(1+y)^{2}}\right) \frac{\partial y}{\partial y_{0}^{\prime}}\left(t, y_{0}, y_{0}^{\prime}\right) d t
$$

Thus, $\partial y / \partial y_{0}^{\prime}\left(x, y_{0}, y_{0}^{\prime}\right)$ cannot vanish identically on $[0, x]$, and so there is a point $x_{0}$ and a neighborhood $\left(x_{0}-\delta, x_{0}+\delta\right)$ in which $\partial y / \partial y_{0}^{\prime}\left(x, y_{0}, y_{0}^{\prime}\right) \neq 0$. The implicit function theorem now implies that $y_{0}^{\prime}=f\left(x, y_{0}\right)$ locally. That is, given $x$, we see that $y_{0}^{\prime}$ is locally a (differentiable) function of $y_{0}$, so that the set $\mathcal{T}_{0}$ is the graph of a function, or this set is a differentiable curve in the plane $\left(y_{0}, y_{0}^{\prime}\right)$ of initial conditions.

Theorem 12 is proved.

Without loss of generality, we restrict ourselves to the case where $y_{0}>-1$ and $y_{0}^{\prime}>0$ (the case $y_{0}<-1$ and $y_{0}^{\prime}<0$ being similar). From Theorem 12, we see that the collection $\mathcal{T}_{0}$ of points $\left(y_{0}, y_{0}^{\prime}\right)$ in $\Pi$ such that $y(x)$ is asymptotically constant at infinity is the graph of a function defined on $(-1, \infty)$. We now show how to estimate this curve.

Use of Lemma 16 and Lemma 13 shows that the curve $\mathcal{T}_{0}$ has a vertical tangent line at $x=-1$, while Lemma 15 can be used to estimate this curve as follows: Lemma 16 tells us that for large $x$, the values of $y^{\prime}(x)$ are exponentially small. Thus, differentiating the representation $y(x)=\sum_{n=0}^{\infty} c_{n}\left(y_{0}, y_{0}^{\prime}\right) x^{n}$, with respect to $x$ and setting the resulting series equal to zero for suitable $x$-ranges we get, solving for $y_{0}^{\prime}$ in terms of $y_{0}$ and stopping after the third term,

$$
y_{0}^{\prime}=\frac{1}{4}\left(y_{0}+\frac{4}{1+y_{0}}\right) x
$$

For $y_{0}>-1$ and $y_{0}^{\prime}>0$, this curve is displayed below.

Eq. (33) serves as a rough estimate for the curve defined by the set $\mathcal{I}_{0}$. Sharper estimates can be found by collecting more terms from the power series representation given in Lemma 15 and solving for $y_{0}^{\prime}$ (after setting the differentiated sum equal to zero) and piecing together various arcs obtained by varying the $x$-values. The resulting curve (with $x=1$ ) is shown in Fig. 4 . Varying the value of $x$ in (33) can be used to "fit" the curve to solutions obtained numerically. Note that the graph of $y_{0}^{\prime}$ as a function of $y_{0}$ (as displayed in Fig. 4) has a vertical tangent line at $y=-1$, in conformity with the theoretical investigations of Lemma 14. As well, we note that initial conditions "above" the curve lead to solutions that are asymptotically linear (not constant), while points below lead to solutions with finite singularities. Finally, we note that this analysis also can be extended to the left plane defined by $y_{0}<-1$. In this case, we get another piece of the curve above (also given by (33)), shown below alongside the one in Fig. 4. 


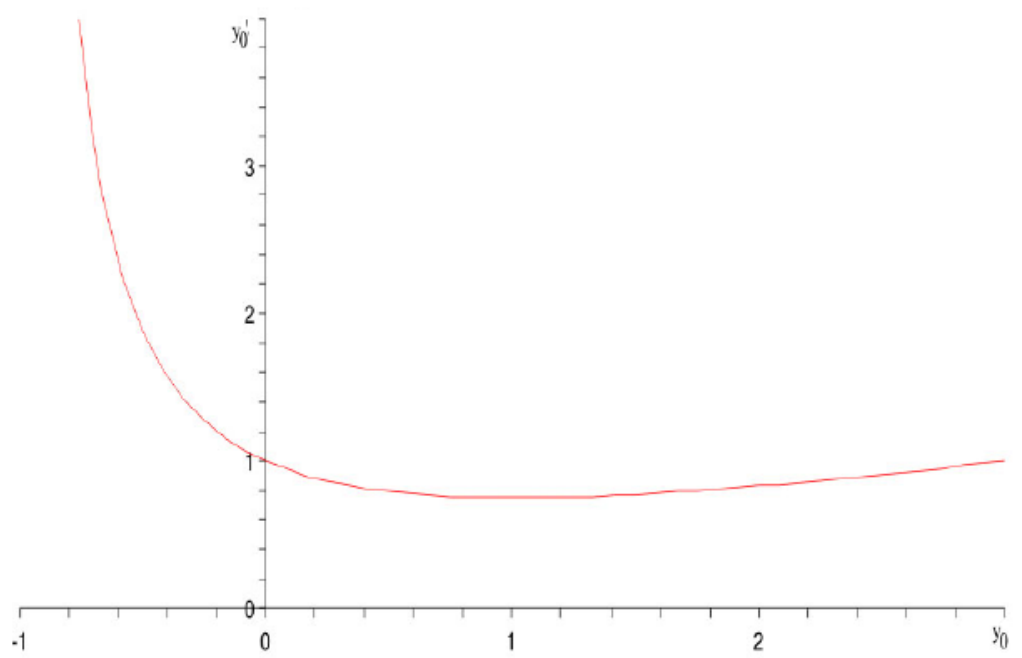

Fig. 4

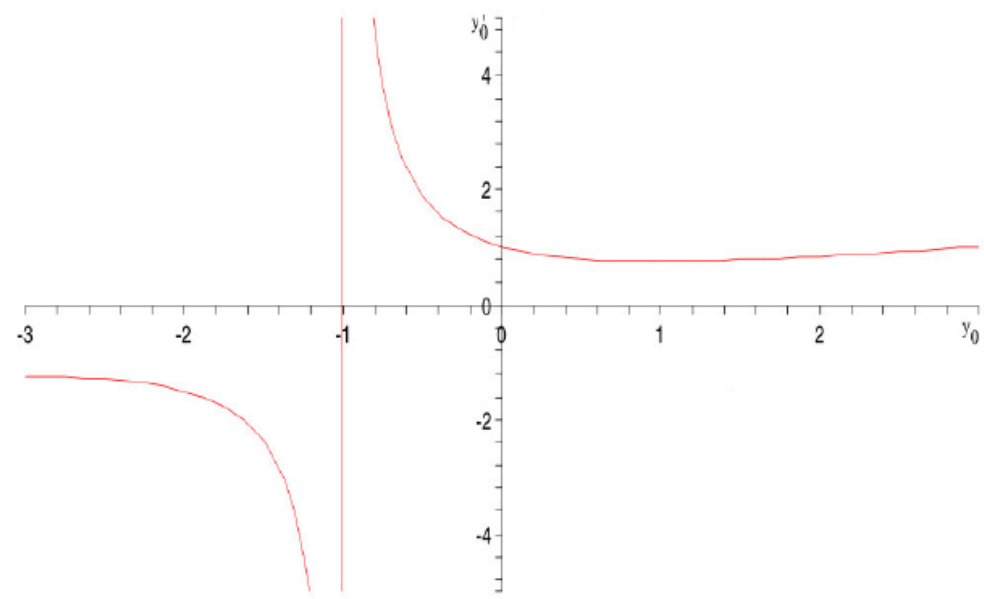

Fig. 5

The two branches of the curve in Fig. 5 approximating the set $\mathcal{T}_{0}$ have the following property:

Theorem 13. Given $y_{0} \neq-1$, there exists a distinguished value $y_{0}^{\prime *}$ of $y_{0}^{\prime}$ (depending on whether $y_{0}>-1$ or $y_{0}<-1$, resp.) such that for all $y_{0}^{\prime}>y_{0}^{\prime *}$ (resp. $\left.y_{0}^{\prime}<y_{0}^{\prime *}\right)$ the solutions are asymptotically linear while for $y_{0}^{\prime}<y_{0}^{\prime *}$ (resp. $\left.y_{0}^{\prime}>y_{0}^{\prime *}\right)$ the solutions have finite singularities. Finally, when $y_{0}^{\prime}=y_{0}^{\prime *}$ the solutions are asymptotically constant.

In order to prove this theorem we need a result that allows us to compare solutions of (1). This is the content of the next lemma.

Lemma 17. Let $y, z$ be any two solutions of (1) such that for some $\delta>0,|1+y(x)|>\delta$ and 
$|1+z(x)|>\delta$ for every $x \in[a, b]$. Then

$$
\left.\left[y^{\prime}(x) z(x)-y(x) z^{\prime}(x)\right]\right|_{a} ^{b}=\int_{a}^{b} \frac{(y(x)-z(x))(1+y(x)+z(x))}{(1+y(x))(1+z(x))} e^{-2 x} d x .
$$

Proof. The argument is Sturmian in nature. Multiply (1) by $z$. With $y$ replaced by $z$ we multiply (1) again by $y$ and subtract the two results. Integrating over $[a, b]$ and simplifying we get

$$
\int_{a}^{b} \frac{(y(x)-z(x))(1+y(x)+z(x))}{(1+y(x))(1+z(x))} e^{-2 x} d x=\int_{a}^{b}\left(y^{\prime \prime}(x) z(x)-y(x) z^{\prime \prime}(x)\right) d x .
$$

Integrating the right-hand side by parts there follows (34).

Lemma 17 is proved.

Proof (Theorem 13). For a given $y_{0}$, the existence of the special initial slope $y_{0}^{\prime *}$ (and corresponding solution, $\left.y^{\prime *}(x)\right)$, is a consequence of Theorem 12.

Case i: Let $y_{0} \geq 0$, a quantity that we now fix, and $y_{0}^{\prime}>0$ (the case $y_{0}^{\prime} \leq 0$ immediately leads to type (4) solutions by Lemma 9). We first show that whenever $y_{0}^{\prime}>y_{0}^{\prime *}$, then the corresponding solution $y(x)$ is asymptotically linear.

For $y_{0}^{\prime}>y_{0}^{\prime *}$, there exits a neighborhood $[0, \eta], \eta>0$, such that for the resulting solutions, $y^{\prime}(x)>y^{\prime *}(x)$ for all $x \in[0, \eta]$.

Assume that the collection of all such $\eta$ has no finite upper bound. Since $y^{\prime^{*}}(x)$ is defined for all $x$ the same is true of $y^{\prime}(x)$. Thus, $y^{\prime}(x)$ tends to a limit at infinity (recall that all solutions and their derivatives have limits in this case, cf., Theorem 6). However, this limit cannot be zero at infinity as this would violate Theorem 12 (by creating yet another asymptotically constant solution for the same $y_{0}$ ). Thus, using the proofs of Lemma 6 and Lemma 8, $y^{\prime}(x)$ tends to a non-zero limit at infinity, and so the solution must be asymptotically linear.

If the collection of these $\eta$ admits a finite upper bound, then there exists a smallest value $\eta \equiv x_{0}>0$ such that $y^{\prime}\left(x_{0}\right)=y^{\prime *}\left(x_{0}\right)$ and $y^{\prime}(x)>y^{\prime *}(x)$ for all $x \in\left[0, x_{0}\right)$. Since $y(0)=y^{*}(0)$ by hypothesis, we deduce from the preceding inequality that $y(x)>y^{*}(x)$ for every $x \in\left[0, x_{0}\right]$. We now use Lemma 17 above with $z(x) \equiv y^{*}(x)$ over $[a, b] \equiv\left[0, x_{0}\right]$. Recall that $y^{\prime}\left(x_{0}\right)=$ $=y^{\prime *}\left(x_{0}\right)=z^{\prime}\left(x_{0}\right)$ and $y^{\prime}(0)>y^{\prime *}(0)=z^{\prime}(0)$ (by hypothesis). Thus,

$$
\begin{aligned}
\int_{0}^{x_{0}} \frac{(y(x)-z(x))(1+y(x)+z(x))}{(1+y(x))(1+z(x))} e^{-2 x} d x & =\left.\left[y^{\prime}(x) z(x)-y(x) z^{\prime}(x)\right]\right|_{0} ^{x_{0}}= \\
& =y^{\prime}\left(x_{0}\right)\left(z\left(x_{0}\right)-y\left(x_{0}\right)\right)-y(0)\left(y^{\prime}(0)-z^{\prime}(0)\right)<0
\end{aligned}
$$

since $z\left(x_{0}\right)-y\left(x_{0}\right)<0, y^{\prime}\left(x_{0}\right)>0$ and $y(0)\left(y^{\prime}(0)-z^{\prime}(0)\right) \geq 0$. This, however, contradicts the fact that the left-hand side is positive since $y(x)>z(x)$ over $\left[0, x_{0}\right)$ and all the other terms are positive. Thus $x_{0}$ cannot exist and so we conclude, from the preceding paragraph, that $y(x)$ is asymptotically linear. 
Let $y_{0}^{\prime}<y_{0}^{\prime *}$. We wish to show that $y$ is a type (4) solution. Arguing as in the previous case, on the assumption that $y(x)$ is defined for all $x$, we cannot have $y^{\prime}(x)$ tend to zero at infinity. Next, we know that the assumption $y_{0}^{\prime} \leq 0$ immediately leads to type (4) solutions by Lemma 9 . Hence we need only consider the case where $y_{0}^{\prime}>0$.

Should $y$ not be of type (4), then it must be asymptotically linear (by one of Lemmas 6-9, as the case may be, depending on the sign of $\left.y_{0}^{\prime}\right)$. We show presently that this is impossible.

For $y_{0}^{\prime}<y_{0}^{\prime *}$, the resulting solution $y^{\prime}(x)<y^{\prime *}(x)$ for all $x \in[0, \eta]$ where $\eta>0$. Since $y(x)$ is asymptotically linear we have $y^{\prime}(x) \rightarrow \beta$ as $x \rightarrow \infty$ where $\beta>0$ (note that $\beta<0$ leads to a type (4) solution since $\left.y_{0}>-1\right)$. Writing $g(x)=y^{\prime}(x)-y^{\prime^{*}}(x)$ for all $x$, we see that $g$ is differentiable and $g(0)<0$, by hypothesis. In addition, since $y^{*}(x)$ is asymptotically constant, for $0<\varepsilon<\beta / 4$ there is an $X_{0}$ such that $\left|y^{\prime *}(x)\right|<\beta / 4$ for all $x>X_{0}$. Furthermore, since $y^{\prime}(x) \rightarrow \beta$ as $x \rightarrow \infty$, there is an $X_{1}>X_{0}$ such that for every $x>X_{1}, y^{\prime}(x)>\beta / 2$. Hence, for $x>X_{1}$, we have $g(x)>\beta / 2-\beta / 4=\beta / 2>0$. But this means that $g\left(x_{0}\right)=0$ for some $x_{0} \in\left[0, X_{1}\right]$, that is, $y^{\prime}\left(x_{0}\right)=y^{\prime^{\prime}}\left(x_{0}\right)$. Letting $x_{0}$ be the smallest such point, if necessary, we can assume that $y^{\prime}(x)<y^{\prime *}(x)$ for all $x \in\left[0, x_{0}\right)$. It follows from this that $y(x)<y^{*}(x)$ for every $x \in\left[0, x_{0}\right)$. As in the previous case we use Lemma 17 over the interval $\left[0, x_{0}\right]$ with $z(x) \equiv y^{*}(x)$. In this case we note that the left-hand side of (35) is negative while the righthand side is positive, leading once again to a contradiction. Hence $y(x)$ cannot be asymptotically linear and so must be of type (4).

Case ii: Finally, let $-1<y_{0}<0$. The case where $y_{0}^{\prime} \leq 0$ leads to type (4) solutions by Lemma 9 and so we can assume that $y_{0}^{\prime}>0$.

Let $0<y_{0}^{\prime}<y_{0}^{\prime *}$. One must prove that the resulting solution $y$ is a type (4) solution. As in the previous cases, if $y(x)$ is defined for all $x$, we cannot have $y^{\prime}(x) \rightarrow 0$ at infinity. On the other hand, $y^{\prime}(x) \rightarrow \beta$, and $\beta<0$ is impossible unless this is actually a type (4) solution. If $y$ is not a type (4) solution, then it follows that $y(x)$ must be asymptotically linear at infinity in the sense that, for some $\beta>0$, we have $y^{\prime}(x) \rightarrow \beta$ as $x \rightarrow \infty$. Repeating the discussion above regarding the $g$-function, there exists a smallest point $x_{0}$ such that $y^{\prime}\left(x_{0}\right)=y^{\prime *}\left(x_{0}\right)$. Because of this, one can assume that $y^{\prime}(x)<y^{\prime^{*}}(x)$ for all $x \in\left[0, x_{0}\right)$, and $y^{\prime}(x)>y^{\prime^{*}}(x)$ for all $x \in\left(x_{0}, \infty\right)$ since the derivatives are each monotone functions. So $y(x)>y^{*}(x)$ for $x>x_{0}$.

Choose $x_{1}>x_{0}$ so large that $y\left(x_{1}\right)>0, y\left(x_{1}\right)+z\left(x_{1}\right)>0$, and $y^{\prime}\left(x_{1}\right)-z^{\prime}\left(x_{1}\right)>0$. This is always possible because of the asymptotic nature of these solutions. We now use (34), with $z(x) \equiv y^{*}(x)$ again, over the interval $[a, b]=\left[x_{1}, X\right]$ where $X$ is chosen so that $y^{\prime}(X)>\beta / 2$. Consider the function $w(x) \equiv 1+y(x)+z(x)$ for $x \geq x_{1}$. Since $y, z$ are each monotone, so is $w$. In addition, $w(x) \geq w\left(x_{1}\right)=1+y\left(x_{1}\right)+z\left(x_{1}\right)>0$, for every $x>x_{1}$. It now follows that the left-hand side of (34) is positive while the right-hand side is negative. This contradiction shows that $y$ is necessarily a type (4) solution. The case $y_{0}<-1$ is similar and is left to the reader.

Theorem 13 is proved.

4.1. Concluding remarks and open questions. For $x<0$ it is not difficult to show that every solution of (1) with initial conditions $y_{0}>-1, y_{0}^{\prime}>0$ or $y_{0}<-1, y_{0}^{\prime}<0$ must have a finite singularity. We sketch the proof for completeness. The change of variable $x=-t, y(x)=u(t)$ sends $(1)$ on $(-\infty, 0]$ into the equation

$$
u^{\prime \prime}+\frac{e^{t}}{4} u+\frac{e^{2 t}}{1+u}=0, \quad t \in[0, \infty)
$$

Since we are dealing with solutions having finite singularities, it suffices to consider initial condi- 
tions for this equation such that $u_{0}>-1, u_{0}^{\prime}<0$ or $u_{0}<-1, u_{0}^{\prime}>0$ since otherwise we already know that the other initial conditions lead to type (4) solutions for $x>0$ by Lemmas $7-$ 9 in the discussion above. We now argue as in the proofs of Lemmas 6-9.

Let $u_{0}>-1, u_{0}^{\prime}<0$. Then there is a right-neighborhood of $t=0$ in which $u(t)<0$, $u^{\prime}(t)<0$ and $u^{\prime \prime}(t)<0$. This then leads to a type (4) solution (as in Lemma 10). On the other hand, if $u_{0}<-1, u_{0}^{\prime}>0$, then there is a right-neighborhood of $t=0$ such that $u(t)<-1$, $u^{\prime}(t)>0, u^{\prime \prime}(t)>0$, and this leads to a type (4) solution (by the methods of Lemma 10). Combining these results we obtain the following theorem.

Theorem 14. Every solution of (1) on $\mathbb{R}$ has a finite singularity.

Placing the pole in (1) at $y=-a, a>0$, leads to no further generality, as this amounts to a rescaling with $e^{-2 x}$ replaced by $e^{-2 x} / 2 a$. The techniques here can be adapted to the cases where

$$
y^{\prime \prime}+f(x) y+\frac{g(x)}{1+y}=0, \quad x \in[0, \infty),
$$

on the assumption that $f, g$ are positive, of exponential order, $f(0)<4 g(0)$, in addition to the usual analyticity requirements. Indeed, more general criteria for a corresponding Theorem 6 can be formulated similar to those described in [10] by restricting the classes of $F$.

We note the asymptotic behavior of those solutions with finite singularities in Subsection 2.1. Numerical considerations indicate that this approach to the value -1 is actually very slow, in conformity with the square root of the negative of the logarithm seen above. We also note that this reveals the nature of the logarithmic branch point. A thorough study of this equation for complex $x$ may reveal more interesting facts about the general solution.

1. Ablowitz M. J., Clarkson P. A. Solitons, nonlinear evolution equations and inverse scattering. - Cambridge, UK: Cambridge Univ. Press, 1999.

2. Ablowitz M.J., Ramani A., Segur H. A connection between nonlinear evolution equations and ordinary differential equations of P-type. I // J. Math. Phys. - 1980. - 21. - P. 715-721.

3. Agarwal R. P., O'Regan D. A note on a singular integral equation arising in a problem in communications // Z. angew. Math. und Mech. - 2001. - 81. - P. 499-504.

4. Agarwal R. P., O'Regan D. Volterra integral equations: the singular case // Hokkaido Math. J. - 2003. - 32. - P. $371-381$.

5. Agarwal R. P., O'Regan D. Fredholm and Volterra integral equations with integrable singularities // Hokkaido Math. J. - 2004. - 33. - P. $443-456$.

6. Alves C. O., Goncalves J. V., Maia L. A. Singular nonlinear elliptic equations in $\mathbb{R}^{n} / /$ Abstract and Appl. Anal. - 1998. - 3, № 3-4. - P. 411-423.

7. Borwein J. M., Lewis A.S., Nussbaum R. D. Entropy minimization, DAD problems, and doubly stochastic kernels // J. Funct. Anal. - 1994. - 123, № 2. - P. 264 -307.

8. Bushell P.J. Class of Volterra and Fredholm nonlinear integral equations // Math. Proc. Cambridge Phil. Soc. - 1976. - 79. - P. 329-335.

9. Constantin A. Positive solutions of quasilinear elliptic equations // J. Math. Anal. and Appl. - 1997. - 213. - P. $334-339$.

10. Dubé S.G.A., Mingarelli A. B. Note on a non-oscillation theorem of Atkinson // Electron. J. Different. Equat. - 2004. - 2004, № 22. - P. $1-6$.

11. Gomes S. M. On a singular nonlinear elliptic problem // SIAM J. Math. Anal. — 1986. — 17, № 6. - P. 13591369.

12. Hale J. K. Ordinary differential equations. - New York: Wiley Int. Publ., 1969. 
13. Hale J. K., Onuchic N. On the asymptotic behavior of solutions of a class of differential equations // Contrib. Different. Equat. - 1963. - 1. - P. $61-75$.

14. Hartman P., Onuchic N. On the asymptotic integration of ordinary differential equations // Pacif. J. Math. 1963. - 13. - P. $1193-1207$.

15. Hille E. Lectures on ordinary differential equations. - New York: Addison-Wesley, 1969.

16. Ince E. L. Ordinary differential equations. - New York: Dover Publ., 1956.

17. Jin Z. R. Solutions for a class of singular semilinear elliptic equations // Nonlinear Anal. - 1998. - 31, № 3 -4. - P. 475 - 492 .

18. Karlin S., Nirenberg L. On a theorem of P. Nowosad // J. Math. Anal. and Appl. - 1967. - 17. - P. $61-67$.

19. Kartsatos A. G. Recent results on oscillation of solutions of forced and perturbed nonlinear differential equations of even order // Stability of Dynamical Systems - Theory and Applications / Ed. J. R. Graef (Lect. Notes Pure and Appl. Math.). - 1977. - 28. - P. 17-72.

20. Michelli C.A., Olsen P. Penalized maximum-likelihood estimation, the Baum-Welch algorithm, diagonal balancing of symmetric matrices and applications to training acoustic data // J. Comput. Appl. Math. - 2000. - 119, № 1-2. - P. 301-331.

21. Nasr A. H. Necessary and sufficient conditions for the oscillation of forced nonlinear second order differential equations with delayed argument // J. Math. Anal. and Appl. - 1997. - 212. - P. 51 - 59.

22. Nowosad P. On the integral equation $\kappa f=1 / f$ arising in a problem in communication // J. Math. Anal. and Appl. - 1966. - 14. - P. 484-492.

23. Nussbaum R. D. Iterated nonlinear maps and Hilbert projective metric, 2 // Mem. Amer. Math. Soc. - 1989. - 79, № 401. - iv + 118 p.

24. Nussbaum R. D. Entropy minimization, Hilbert projective metric, and scaling integral-kernels // J. Funct. Anal. - 1993. - 115, № 1. - P. 45 - 99.

25. Nussbaum R. D. Hilbert's projective metric and iterated nonlinear maps // Mem. Amer. Math. Soc. - 1988. - 75, № 391. - iv+137 p.

26. Parker G. E., Walters T.J. Constructing solutions to $\int_{0}^{1} f(j) A(j, x) / f(x) d j=\int_{0}^{1} f(x) A(x, j) / f(j) d j / /$ SIAM J. Math. Anal. - 1982. - 13, № 5. - P. 856-865.

27. Swanson C. A. Comparison and oscillation theory of linear differential equations. - New York: Acad. Press, 1968.

28. Yin Z. Monotone positive solutions of second order nonlinear differential equations // Nonlinear Anal. 2003. - 54. - P. $391-403$.

29. Walters T. J., Parker G. E. Positive nonlinear integral equations with reciprocals of the solution in the integrand // Nonlinear Anal. - 1981. - 5, № 11. - P. 1163-1172.

30. Wang J., Gao W., Zhang Z. Singular nonlinear boundary value problems arising in boundary layer theory // J. Math. Anal. and Appl. - 1999. - 233. - P. 246-256.

31. Zhao Z. Positive solutions of nonlinear second order ordinary differential equations // Proc. Amer. Math. Soc. -1994 . - 121, № 2. - P. 465-469. 